\title{
Primary resistance to EGFR inhibition in glioblastoma is mediated by a TNF-JNK-AxI-ERK signaling axis
}

\author{
Gao Guo ${ }^{1}$, Ke Gong ${ }^{1}$, Sonia Ali ${ }^{1}$, Neha Ali ${ }^{1}$, Shahzad Shallwani ${ }^{1}$, Kimmo J. Hatanpaa ${ }^{2}$, \\ Edward Pan ${ }^{1}$, Bruce Mickey ${ }^{3}$, Sandeep Burma ${ }^{4}$, David H. Wang ${ }^{5,6}$, Santosh Kesari ${ }^{7}$, Jann N \\ Sarkaria $^{8}$, Dawen Zhao ${ }^{9}$, and Amyn A. Habib ${ }^{1,6,{ }^{*}}$ \\ ${ }^{1}$ Department of Neurology and Neurotherapeutics, University of Texas Southwestern Medical \\ Center, Dallas TX 75390 \\ ${ }^{2}$ Department of Pathology, University of Texas Southwestern Medical Center, Dallas TX 75390 \\ ${ }^{3}$ Department of Neurosurgery, University of Texas Southwestern Medical Center, Dallas TX \\ 75390 \\ ${ }^{4}$ Department of Radiation Oncology, University of Texas Southwestern Medical Center, Dallas TX \\ 75390 \\ ${ }^{5}$ Department of Internal Medicine at the University of Texas Southwestern Medical Center, Dallas \\ TX 75390, \\ ${ }^{6}$ VA North Texas Health Care System, Dallas TX 75216 \\ ${ }^{7}$ Department of Translational Neuro-Oncology and Neurotherapeutics, John Wayne Cancer \\ Institute at Providence Saint John's Health Center, Santa Monica, CA 90404 \\ ${ }^{8}$ Department of Radiation Oncology, Mayo Clinic, Rochester, MN 55905, USA \\ ${ }^{9}$ Departments of Biomedical Engineering and Cancer Biology, Wake Forest School of Medicine, \\ Winston Salem, NC 27157
}

\begin{abstract}
Aberrant EGFR signaling is widespread in cancer, making the EGFR an important target for therapy. EGFR gene amplification and mutation are common in glioblastoma (GBM), but EGFR inhibition has not been effective in treating this tumor. Here, we propose that primary resistance to EGFR inhibition in glioma cells results from a rapid compensatory response to EGFR inhibition that mediates cell survival. We show that in glioma cells expressing either EGFR wild type or the mutant EGFRvIII, EGFR inhibition triggers a rapid adaptive response driven by increased TNF
\end{abstract}

\footnotetext{
Users may view, print, copy, and download text and data-mine the content in such documents, for the purposes of academic research, subject always to the full Conditions of use: http://www.nature.com/authors/editorial_policies/license.html\#terms

*To whom correspondence should be addressed at: University of Texas Southwestern Medical Center, North Texas VA Healthcare System, Mail Code 151, 4500 South Lancaster Road, Dallas TX 75216, Phone: (214) 857-3610, Amyn.Habib@UTSouthwestern.edu. Author Contributions:

AH, GG and KG designed experiments. GG, KG, SA, NA, SS, and DHW performed or assisted with experiments. BM, KH, JS, and SK provided tumor samples. EP, SB and DZ, JS, GG and AH analyzed data. AH and GG wrote the manuscript with contributions from KG, EP and KH. Study supervision: AH.

Competing Financial Interests Statement

The authors declare no competing financial interests.
} 
secretion that leads to activation of a TNF-JNK-Axl-ERK signaling axis. Inhibition of this adaptive axis at multiple nodes renders glioma cells with primary resistance sensitive to EGFR inhibition. Our findings provide a possible explanation for the multiple failures of anti-EGFR therapy in GBM and suggest a new approach to the treatment of EGFR expressing GBM using a combination of EGFR and TNF inhibition.

\section{Keywords}

Erlotinib; Resistance; glioblastoma; Axl; ERK; JNK; primary resistance; tumor necrosis factor; TNF; EGFR inhibition; GBM

\section{Introduction}

The identification of genetic abnormalities that are specific to cancer cells has made it possible to develop targeted treatments. The EGFR is a prime target in this therapeutic approach, since it is overexpressed in many types of cancers and may be a key driver of the malignant phenotype. An exciting development in recent years was the identification of EGFR activating mutations in a significant subset of lung cancers, that render cells harboring such mutations to become oncogene addicted and very sensitive to the effects of EGFR tyrosine kinase inhibitors (TKIs) ${ }^{1,2}$. However, the inevitable development of secondary resistance has limited the effectiveness of EGFR inhibition in lung cancer. The development of secondary resistance in lung cancer has spurred intense investigation into mechanisms of EGFR TKI resistance and resulted in important insights into secondary resistance to EGFR TKIs in lung cancer. The major mechanisms identified in lung cancer include the emergence of EGFR mutations such as the T790M mutation and activation of other receptor tyrosine kinases such as Met or Axl that confer resistance to EGFR TKIs ${ }^{3}$. In addition to genetic and delayed mechanisms, rapid feedback loops with activation of STAT3 have also been invoked to mediated EGFR TKI resistance in lung cancer cells with EGFR activating mutations ${ }^{4,5}$. However, most EGFR expressing tumors both in the lung and the brain do not appear to be oncogene addicted and EGFR TKIs, so far, have not been effective in such cancers.

EGFR gene amplification and increased EGFR expression are detected in 40-50\% GBMs, the most common primary malignant adult brain tumor ${ }^{6,7}$. EGFRvIII is the most common oncogenic EGFR mutant in GBM and may be more sensitive to EGFR inhibition ${ }^{8}$. There has been a substantial, and thus far, unsuccessful effort to inhibit the EGFR as a therapeutic strategy in $\mathrm{GBM}^{9}$. While not much is known about what mediates primary resistance to EGFR inhibition in GBMs expressing EGFR wild type, a number of studies have provided key insights into mechanisms that mediate secondary resistance to erlotinib in EGFRvIII expressing glioma cells after an initial period of responsiveness. For example, prolonged EGFR inhibition leads to an increased expression of PDGFR $\beta$ that mediates a secondary resistance to erlotinib ${ }^{10}$. In another study, it was demonstrated that secondary resistance to erlotinib in GBM is mediated via a dynamic downregulation of EGFRvIII ${ }^{11}$. A comparison of erlotinib sensitivity of lung cancer mutants vs. EGFRvIII suggested that EGFRvIII is resistant to erlotinib because of lower kinase-site occupancy and rapid cycling compared to 
lung cancer mutants ${ }^{12}$. Another study has identified a Urokinase receptor-Bim signaling axis as mediating EGFR inhibitor resistance ${ }^{13}$.

Primary resistance to EGFR inhibition in cancer cells expressing EGFRwt or non responsive EGFR mutants may occur because the EGFR does not drive survival/proliferation of these cells or because adaptive signals prevent cell death. If primary resistance is mediated via an early adaptive response, there is the possibility of inhibiting this adaptive response and overcoming primary resistance to EGFR inhibition. Here, we show that primary resistance in EGFR expressing glioma cells is mediated by a rapid adaptive signaling pathway that is triggered by inhibition of EGFRwt or mutant EGFR. We propose that a TNF-JNK-Axl-ERK signaling pathway mediates this adaptive response. Inhibition of this pathway in EGFR expressing glioma cells confers sensitivity to EGFR inhibition in cell culture as well as in a mouse model.

\section{Results}

\section{Erlotinib induces a feedback activation of ERK in glioma cells}

In order to elucidate mechanisms of EGFR inhibition resistance in glioma cells, we examined signal transduction events following erlotinib exposure in glioma cells. We used EGFR expressing patient derived primary GBM neurospheres as well as established glioblastoma cell lines expressing EGFRwt or EGFRvIII as shown in Figure 1a. Signaling from EGFRvIII is constitutive, while signaling from overexpressed EGFRwt may be constitutive or ligand induced ${ }^{7,14-19}$. GBM9, GBM39 and SK987 neurospheres express EGFRvIII and also EGFRwt and have been described previously ${ }^{20-23}$. We started our investigation by examining activation of Akt, STAT3 and ERK, since these pathways play an important role in cancer cell survival. Exposure of GBM9 neurospheres to erlotinib demonstrated ERK activation in untreated cells that decreased after addition of erlotinib suggesting that EGFR was driving the activation of ERK. A reactivation of ERK is seen at 24-48h in erlotinib treated cells, likely triggered by a feedback mechanism, since the EGFR remains inactivated (Figure 1b). However, we did not detect an increase in Akt or STAT3 activation (Figure 1b). Similar results were found in patient derived primary GBM39 and SK987 cells (Figure 1c-d). We also examined signal transduction in established glioblastoma cell lines U87EGFRwt, U87EGFRvIII and U251EGFRwt and found a similar feedback activation of ERK in response to EGFR inhibition (Figure 1e-f and Supplementary Figure 1a). Also, similar results were found with afatinib, an irreversible inhibitor of EGFR kinase, (Supplementary Figure 1b). If we use a higher concentration of erlotinib, STAT3 and Akt activation are suppressed. However, we do not detect any reactivation of STAT3 or Akt in the presence of continuing EGFR inhibition (Supplementary Figure 1c-g). Thus, of the three pathways examined, only ERK became activated in response to EGFR inhibition. In U87EGFRwt cells, ERK and EGFR activation are similar in serum or serum free conditions (Supplementary Figure 1h). Basal EGFR and ERK activation could be inhibited by Cetuximab, which blocks ligand binding to the EGFR, suggesting autocrine activation of EGFR under serum starved conditions (Supplementary Figure 1i). As expected, further increases in EGFR and ERK activation were detected when exogenous EGF was added (Supplementary Figure 1j). 


\section{Inhibition of AxI blocks EGFR inhibition mediated ERK activation}

Activation of other receptor tyrosine kinases such as Met or Axl has been identified as a major mechanism of secondary resistance to EGFR inhibition in lung cancer cells. In glioma, EGFRvIII expression leads to coactivation of multiple RTKs, such as Met and a combined inhibition of EGFR and Met or Akt/mTOR is more effective than inhibition of the EGFR alone ${ }^{24-29}$. Increased expression of PDGFR $\beta$ has been described in response to prolonged EGFR inhibition in glioma cells ${ }^{10}$. We detected phosphorylation of the RTK Axl following exposure of cells to erlotinib for $48 \mathrm{~h}$ in patient derived GBM neurospheres and in all cell lines examined (Figure 1b-f and Supplemental Figure 1a). Erlotinib also induces activation of Met in established cell lines, but only in one patient derived neurosphere 987 (Figure 1b-f and Supplementary Figure 1a). Since Axl activation is seen in all patient derived neurospheres and established cell lines tested, we focused on Axl in this study.

We examined if Axl was responsible for activation of ERK in response to EGFR inhibition. We used the Axl inhibitor R428 and found that inhibition of Axl results in a block of erlotinib induced ERK activation in patient derived GBM neurospheres as well as in multiple cell lines as shown in Figure 1g-j. Similarly, siRNA knockdown of Axl also results in a block of erlotinib induced ERK activation (Figure 1k-n). Thus, Axl plays an essential role in EGFR induced inhibition of ERK activation.

\section{Activation of JNK by EGFR inhibiton triggers a survival feedback loop}

We next examined whether inhibition of key signaling pathways known to be active in EGFR signaling could block erlotinib induced Axl and ERK activation. We found that the JNK inhibitor SP600125 inhibited erlotinib induced activation of Axl and ERK in patient derived neurospheres and established GBM cell lines, but the p38 MAPK inhibitor SB203580 failed to do so (Figure 2a-d). We also found that siRNA knockdown of JNK 1 and 2 resulted in a block of erlotinib induced EGFR Axl and ERK activation (Figure 2e-g). Furthermore, erlotinib exposure of cells results in activation of JNK as detected by phosphorylation of JNK in Western blots (Figure $2 \mathrm{~h}-\mathrm{k}$ ). These findings suggest that JNK activation plays an essential role in erlotinib induced ERK activation. JNK refers to cJun $\mathrm{NH}_{2}$-terminal kinases that are MAP kinases that phosphorylate c-Jun. This results in activation of the transcription factor AP1. Consistent with data demonstrating JNK activation in response to EGFR inhibition, erlotinib also induces increased activity of the AP-1 reporter (Figure 21). These experiments support a model in which EGFR inhibition leads to the activation of JNK signaling and, in turn, JNK signaling mediates an activation of Axl. Axl activation then leads to ERK activation.

EGFR inhibition has also been reported to result in a rapid activation of NF- $\mathrm{kB}$ in lung cancer cells expressing EGFR activating mutations ${ }^{30}$. However, we did not detect an increase in NF- $\kappa$ B transcriptional activity in response to EGFR inhibition in glioma cells, whereas LPS efficiently activated NF- $\mathrm{BB}$ transcriptional activity (Supplementary Figure 2ab). 


\section{Activation of AxI results from increased expression of GAS6}

Next, we examined the mechanism of Axl activation following exposure to erlotinib. Axl is activated about $24-48$ hours after the EGFR is inhibited. We investigated the possibility that erlotinib may lead to increased expression of GAS6, the ligand for Axl. We examined the erltoinib induced expression of $G A S s 6$ at various time points by real time quantitative PCR. EGFR inhibition leads to an increase in GAS6 in patient derived neurospheres and in established GBM cell lines (Figure 3a-d). Importantly, inhibition of JNK blocks erlotinib induced expression of $G A S 6$ (Figure 3a-d), consistent with the previously noted inhibition of erlotinib induced Axl activation by a chemical inhibition or silencing of JNK. An increased level of GAS6 was confirmed by ELISA and Western blot (Figure 3e-f). These findings suggest that erlotinib induced activation of Axl likely results from increased levels of GAS6. Next, we examined whether activation of JNK leads to increased transcription of GAS6. Using TFBIND program, multiple predicted c-Jun (AP-1) binding sites were identified 500 bp upstream of the putative transcription start site of GAS6 (Figure 3g). We then undertook a chromatin immunoprecipitation (ChIP) assay to investigate the presence of AP-1 at the GAS6 promotor. Erlotinib exposure leads to the binding of cJun to the GAS6 promoter in GBM9 and U87EGFRwt cells as shown in Figure 3h-i.

\section{JNK is activated by an Erlotinib induced increase in TNF levels}

Next, we examined the mechanism of erlotinib-induced JNK activation. Previous studies have found that EGFR inhibition in lung cancer cells results in increased secretion of inflammatory cytokines ${ }^{5}$. We hypothesized that EGFR inhibition in glioma cells may lead to a similar release of cytokines. Since TNF is a known and potent activator of JNK ${ }^{31}$, we examined the level of TNF in patient derived GBM neurospheres as well as EGFR expressing cell lines. Erlotinib leads to a significant and rapid increase in TNF mRNA levels as determined by real time quantitative PCR and shown in Figure 4a-d. The increase in TNF was confirmed at a protein level by ELISA as shown in Figure 4e. Increase in TNF levels was noted in multiple additional lines and patient derived neurospheres as shown in Supplementary Figure 2c-d. Importantly, increased TNF levels can also be induced by EGFR inhibition in tumors growing in mice as shown in Figure 4f. In this experiment patient derived primary GBM9 cells were injected into the flanks of nude mice. After formation of palpable tumor, erlotinib was administered as indicated followed by harvesting of tumors and ELISA for TNF. An erlotinib induced increase in TNF can be detected 1 day after administration of erlotinib (Figure 4f). In addition, we also detect erlotinib induced activation of JNK, Axl and ERK in mouse tumors (Figure 4g), peaking around 2-7 days and subsiding by 14 days.

Next, we examined whether TNF played an essential role in erlotinib induced JNK activation. Indeed, we find that the use of a neutralizing antibody to TNF lead to inhibition of erlotinib-induced JNK activation (Figure 4h). Furthermore, erlotinib-induced Axl and ERK activation were also blocked by TNF inhibition. Similar results were found with siRNA knockdown of TNFR1 (Figure 4i). We also examined the level of TNFR1 in glioma cells treated with erlotinib. We found that erlotinib induces a downregulation of TNFR1 in patient derived samples and established GBM cell lines as shown in Supplementary Figure 
2e. As it is known that TNF induces downregulation of its receptor ${ }^{32}$, this finding provides evidence that TNF signaling is activated by EGFR inhibition.

\section{Inhibition of the TNF-JNK-AxI-ERK axis confers Erlotinib sensitivity to EGFR expressing glioma cells}

To investigate if this TNF-JNK-Axl-ERK signaling pathway influences the biological response to EGFR inhibition, we examined the effect of inhibiting this pathway on the viability of glioma cells exposed to erlotinib. First we examined the sensitivity of EGFR expressing GBM cell lines to erlotinib. The established cell lines appear to be completely resistant to Erlotinib regardless of whether EGFRwt or EGFRvIII is expressed (Figure 5a). Patient derived primary nuerosphere GBM9 and GBM39 cells are resistant to EGFR inhibition at a low concentration of erlotinib (100nM). However, when combined with JNK inhibition using SP600125 or ERK inhibition using U0126, erlotinib leads to a significant cell death, while cells are resistant to JNK or ERK inhibition alone (Figure 5b-c). Inhibitors of multiple other signaling pathways failed to sensitize glioma cells to EGFR inhibition (Supplementary Figure 2f). Established glioblastoma cell lines are also resistant to EGFR inhibition or JNK inhibition or ERK inhibition alone, but a combined inhibition of EGFR with ERK or JNK leads to a significant cell death (Figure 5d-e). A biological inhibition of JNK using siRNA knockdown also renders glioma cells sensitive to EGFR inhibition as shown in Figure 5f-h and Supplementary Figure 2g.

Next, we examined whether inhibition of Axl would also render resistant glioma cells sensitive to EGFR inhibition, since Axl inhibition blocks the erlotinib induced ERK activation. Indeed, as shown in Figure 5i-k, a specific chemical inhibitor of Axl R428 renders patient derived GBM neurospheres and U87EGFRwt cells sensitive to erlotinib. R428 by itself does not affect the viability of these cells. Axl inhibition also confers sensitivity to erlotinib in established GBM cell lines (Figure 5j). Similarly siRNA knockdown of Axl, also confers erlotinib sensitivity to GBM9, GBM39 and U87EGFR cells (Figure $51-n$ and Supplementary Figure 2h).

Finally, we examined if inhibition of TNF signaling could render glioma cells sensitive to erlotinib. Indeed, inhibition of TNF signaling with the use of Etanecept (Enbrel) renders patient derived primary GBM neurospheres as well as established GBM cell lines sensitive to the effects of EGFR inhibition as shown in Figure 6a-c. Similarly, siRNA knockdown of TNFR1 also results in increased sensitivity of cells to EGFR inhibition (Figure 6d-f and Supplementary Figure 2i). Furthermore, the use of thalidomide, an inhibitor of TNF ${ }^{33}$ also had the same effect (Figure 6g-i). Thalidomide may inhibit other cytokines, and we confirmed that thalidomide does indeed block the erlotinib-induced increase in TNF secretion in glioma cell lines (Supplementary Figure 3a-b). We also confirmed that the use of Enbrel or Thalidomide resulted in an interruption of the erlotinib-induced activation of the JNK-Axl-ERK signaling pathway (Figure $6 \mathrm{j}-1$ ). In addition, a neutralizing antibody to TNF also enhanced sensitivity both patient derived and established GBM lines to EGFR inhibition (Supplementary Figure 3c-d). In addition, afatinib, an irreversible inhibitor of EGFR kinase, in combination with thalidomide had a similareffect (Supplementary Figure 
3e-f). Thus, interruption of the adaptive TNF-JNK-Axl-ERK signaling axis at any node, renders resistant glioma cells sensitive to EGFR inhibition.

Conversely, activation of TNF signaling by addition of exogenous TNF, results in protection from cell death induced by EGFR inhibition in patient derived primary GBM neurospheres. In this experiment, we used a erlotinib concentration of $1 \mu \mathrm{M}$ which induces a significant cell death in these cells. Addition of exogenous TNF protects patient derived primary GBM9 and GBM39 from cell death induced by EGFR inhibition (Figure 6m-n).

Changes in cell viability were also measured by Flow Cytometry for Annexin V and Caspase 3/7 activation (Supplementary Figures 4-7). The data show that glioma cells are undergoing apoptotic cell death when erlotinib is used in combination with Axl, JNK, ERK or TNF inhibition. Finally, cell proliferation was examined by using a cell counting assay. Erlotinib was used in combination with Axl, JNK, ERK or TNF inhibition and the results are shown in Supplementary Figure 8, showing significant decreases in cell numbers in response to combined inhibition of EGFR and JNK, Axl or ERK.

\section{Inhibiting the adaptive response renders primarily resistant glioma cells sensitive in a mouse model}

Erlotinib-induced JNK activation appears to play a central role in orchestrating the adaptive response underlying primary resistance of glioma cells to EGFR inhibition. Thus, we examined the effect of inhibiting JNK in a xenograft model of GBM in athymic mice. The experiment was conducted by injecting patient derived GBM9 neurospheres in the flanks of athymic mice. Once subcutaneous tumors became visible, the mice were divided into control, erlotinib alone, JNK inhibitor (SP600125), or erlotinib+SP600125. erlotinib was administered to animals by oral gavage $\left(50 \mathrm{mg} / \mathrm{kg}\right.$ ) ${ }^{23}$ for 10 days and SP6001125 was administered at a concentration of $40 \mathrm{mg} / \mathrm{kg}$ i.p. daily for 10 days ${ }^{34}$. As is shown in Figure $7 \mathrm{a}$, a combined inhibition of JNK and EGFR strongly inhibits the growth of tumors, whereas SP600125 or erlotinib alone has no significant effect on the growth of tumors.

Next, we undertook an experiment to examine the effect of a combined inhibition of TNF and EGFR. We used thalidomide to inhibit TNF, because thalidomide, is known to penetrate the blood brain barrier and has been previously used in GBM (although not in combination with EGFR inhibition) ${ }^{35}$. The experiment was conducted by injecting patient derived GBM9 neurospheres in the flanks of athymic mice. Once subcutaneous tumors became visible, the mice were divided into control, erlotinib alone, thalidomide alone, or erlotinib +thalidomide. Erlotinib was administered to animals by oral gavage $(50 \mathrm{mg} / \mathrm{kg}$ ) daily for 10 days and thalidomide was administered at a concentration of $150 \mathrm{mg} / \mathrm{kg}$ i.p. daily for 10 days ${ }^{34,36}$. As is shown in Figure 7b, a combined inhibition of TNF and EGFR strongly inhibits the growth of tumors, whereas thalidomide or erlotinib alone has no significant effect on the growth of tumors.

Next we undertook an orthotopic experiment. Patient derived GBM9 cells were implanted intracranially followed by bioluminescence imaging. As we have described recently, GBM9 neurospheres rapidly form tumors in an intracranial model ${ }^{20}$. When tumors became visible on bioluminescence imaging, the mice were divided into four groups and treated with 
control gavage, erlotinib alone, thalidomide alone, or a combination of erlotinib and thalidomide. While neither erlotinib nor thalidomide alone had a significant effect, the combined treatment resulted in a highly significant improved survival of mice treated with a combination of EGFR and TNF inhibition (Figure 7c-d). The orthotopic experiment was repeated with afatinib, another EGFR inhibitor with similar results as shown in Supplementary Figure 9a-b. In our animal experiments, erlotinib or afatinib was used in combination with thalidomide or SP600125 for a total of 10 days without significant immediate short term toxicity up to the time the mice were sacrificed. The effects of these interventions on the body weight of mice are shown in Supplemental Figure 9c-f.

Activation of the JNK-Axl-ERK signaling axis can be detected in intracranial tumors in mice exposed to erlotinib for various time points by Western blot as shown in Figure 7e-f. We also examined the effect of therapeutic intervention and found that use of a TNF inhibitor (thalidomide) blocked the EGFR inhibition induced upregulation of the TNF-JNKAxl-ERK signaling axis in intracranial tumors. (Figure 7g). The temporal profile of this activation is similar to what was noted in the subcutaneous model (Figure $4 \mathrm{~g}$ ) and shows an increased activation of this pathway peaking at 2-7 days and subsiding by 14 days. We also examined activation of JNK, Axl and ERK by IHC as shown in Supplementary Figure 10. The results are consistent with our Western blot results and show that EGFR inhibition leads to activation of the JNK-Axl-ERK signaling axis and administration of a TNF inhibitor (thalidomide) blocks activation of this pathway.

\section{Discussion}

The major finding in our study is that EGFR inhibition in glioma cells results in triggering of a rapid adaptive response that mediates resistance to EGFR inhibition. If this adaptive response is blocked, glioma cells with primary or intrinsic resistance become sensitive to EGFR inhibition and undergo cell death following cessation of EGFR signaling. Previous studies have shown that inhibition of specific tyrosine kinases or key downstream signals in cancer cells leads to a feedback mediated escape from pathway inhibition by reprogramming of signaling pathways that frequently lead to a resumption of previously suppressed signals or activation of alternative signals that are functionally similar ${ }^{37,38}$. Thus, the cancer cell tries to maintain homeostasis and it is likely that such mechanisms are an important contributor to drug resistance. Our study demonstrates that a TNF-JNK-Axl-ERK signaling axis mediates an adaptive response to EGFR inhibition in glioma cells. is triggered in response to EGFR inhibition. Inhibition of this adaptive response in combination with EGFR inhibition is effective in treatment of glioma tumors in a mouse model.

EGFR gene amplification and overexpression are found in 40-50\% of GBMs and about half of these tumors express the constitutively active oncogenic mutant EGFRvIII ${ }^{7,39}$. EGFRvIII does not bind ligand and is considered constitutively active, although recent studies have revealed a role of co-expressed EGFRwt in the activation of EGFRvIII ${ }^{40,41}$. A number of studies have demonstrated that EGFRvIII has a greater oncogenic potential compared to EGFRwt ${ }^{39}$. Recent studies have provided key insights into EGFRvIII downstream signaling and elucidated a role of STAT3 as a key downstream signal ${ }^{41-43}$. However, EGFRwt may also have an oncogenic role in GBM ${ }^{16}$. Activation of EGFRwt may be mediated by co- 
expression of EGFR ligands in the tumor and a number of studies have documented the presence of EGFR ligands in GBM ${ }^{14,18}$. In addition, overexpression of EGFRwt may also result in a ligand-independent constitutive signaling $14,15,17,18$. Thus, EGFR signaling is likely to be active in GBM cells that express either constitutively active EGFR mutants or EGFRwt. Furthermore, EGFR signaling in patient derived primary GBM neurospheres as well as in established GBM cell lines appears to have a role in cell survival, since EGFR inhibition in combination with a block of the TNF-JNK-Axl-ERK adaptive response results in cell death in both patient derived primary GBM cells and in established GBM cell lines. Inhibition of the TNF-JNK-Axl-ERK axis in the absence of EGFR inhibition has no significant effect on GBM cell viability.

Multiple efforts to inhibit the EGFR have failed in $\mathrm{GBM}^{9}$, the most recent being the failure of a vaccine against EGFRvIII. The first study of targeted EGFR TKI in GBMs involved a single-arm phase II trial of gefitinib in GBM patients at first recurrence. No radiographic responses were noted and median overall survival (OS) was about 39 weeks ${ }^{44}$. Subsequent studies with erlotinib in recurrent GBM patients were also not effective. Trials of EGFR TKIs in combination with temozolomide and XRT in the newly-diagnosed GBM patient population have not demonstrated significantly improved outcomes 45,46 . Another treatment strategy involves targeting the EGFRvIII mutation, with a vaccine (rindopepimut) conjugated to keyhole limpet hemocyanin (KLH) designed to generate a specific immune response against tumor cells with EGFRvIII mutations. A phase II multicenter trial of rindopepimut in newly-diagnosed GBM patients demonstrated median OS of 21.8 months and 3 -year survival of $26 \%$, suggesting some early promise of efficacy ${ }^{47}$. However, interim analysis of the subsequent phase III study of rindopepimut plus temozolomide in newlydiagnosed GBM patients (ACT IV) demonstrated no difference in median OS compared to temozolomide plus control KLH injections, and thus the trial was discontinued early (Clinicaltrials.gov identifier: NCT01480479).

The dynamic complexity of the glioblastoma genome poses a formidable challenge to effective treatment. Tumor heterogeneity may also limit the effectiveness of EGFR inhibition ${ }^{48,49}$. We propose that the multiple failures of anti-EGFR therapy in GBM may have resulted, at least in part because of a rapid adaptive response triggered by an increase in TNF signaling. TNF is a central player in the inflammatory response and also in the pathogenesis of cancer. Depending on the cellular context, TNF signaling may promote cell survival or cell death. Our findings indicate that TNF is the primary mediator of intrinsic resistance to EGFR inhibition and promotes cell survival in response to a loss of EGFR signaling via activation of JNK. JNK, in turn, increases expression of GAS6, a ligand for the Axl receptor. Inhibition of TNF or JNK blocks Erlotinib induced Axl activaton. Axl activation, in turn leads to ERK activation. Interruption of the TNF-JNK-Axl-ERK axis at any node results in increased sensitivity to EGFR inhibition. Our data that in GBM xenograft tumors, erlotinib induces an upregulation of TNF in about 24 hours and the TNF level subsides in about a week, while the JNK-ERK-Axl activation subsides in about 2 weeks. It is thus not feasible to detect EGFR inhibition-induced TNF upregulation or JNK-ERK-Axl activation in archival tissue from patients following erlotinib treatment since tissue is not sampled at early time points following TKI treatment. It is likely that key mechanisms of secondary resistance such as a downregulation of EGFRvIII or upregulation of PDGFRB 
also contribute to the failure of anti-EGFR treatment ${ }^{10,11}$. Similarly, a Urokinase receptorBim signaling axis may also contribute to EGFR inhibition resistance ${ }^{13}$.

GBM is a devastating and intractable disease. Temozolomide is the first line chemotherapy drug used in GBM, and in combination with surgery and radiation, results in a modest increase in overall survival of patients ${ }^{50}$. No targeted treatment has proven effective in GBM. Our data indicate that EGFR inhibition may be effective, if combined with an inhibition of a component of the TNF-JNK-Axl-ERK signaling axis. Remarkably, the combined inhibition of EGFR and TNF-pathway is effective even in established GBM cell lines which are otherwise completely resistant to EGFR inhibition. The identification of this TNF-JNK-Axl-ERK signaling axis suggests that it may be possible to target and inhibit this adaptive response at multiple nodes, alone or in combination. We have found that EGFR inhibition in conjunction with JNK inhibition is effective in an animal model. Additionally, a combination of EGFR inhibition and thalidomide is also very effective in suppressing the growth of GBM tumors in both a subcutaneous as well as an intracranial animal model. Thalidomide crosses the blood brain barrier and, indeed, has been previously used clinically in GBM without success ${ }^{35}$. This is consistent with our results that show no effect of thalidomide in the absence of EGFR inhibition. Importantly, this approach is effective in EGFRwt as well as mutant EGFRvIII expressing tumors. EGFR expression is detected in the majority of GBMs and thus this approach could be broadly applicable in this disease.

Furthermore, a rapid translation of these findings to the clinic is possible, given the wealth of TNF inhibiting drugs and biologicals in clinical practice, including drugs such as thalidomide that penetrate the blood brain barrier.

\section{Online Methods}

\section{Plasmids, transfection and generation of Cell lines}

Primary GBM neurosphere cultures were generated directly from human GBM tumor specimens. Cells were cultured in DMEM F12 supplemented with B27 without Vitamin A, and with EGF (20ng/ml) and bFGF (20 ng/ml). GBM9 was provided by Dr. James Van Brocklyn and GBM39 was provided by Dr. C. David James via Dr. Frank Furnari. U87EGFRwt and U87EGFRvIII cells were provided by Dr. Frank Furnari and have been described previously ${ }^{48}$. U251MG expressing EGFRwt were generated as we have described previously ${ }^{14}$. Cell lines were authenticated using a Promega StemElite ID system which is an STR based assay. NF- $\kappa B-L U C$ plasmid was provided by Dr. Ezra Burstein (UT Southwestern). The 3xAP1pGL3 reporter plasmid was obtained from Addgene (\#40342).

\section{Luciferase assays}

Cells were plated in 48 well dishes to $70 \%-80 \%$ confluence followed by with NF- $\kappa B-L U C$ or 3xAP1pGL3 reporter plasmid using lipofectamine 2000. A dual-luciferase reporter assay system was used according to manufacturer's instructions (Promega, Madison WI). Firefly luciferase activity was measured in a luminometer and normalized on the basis of Renilla luciferase activity.. Experiments were done in triplicate and 3 independent experiments were done. 


\section{RNA interference}

For transient silencing we used a pool of siRNA sequences directed against human TNFR1, Axl, JNK1, JNK2 or control (scrambled) obtained from Santa Cruz. siRNAs were introduced into cells using the Lipofectamine2000 (Invitrogen Carlsbad, CA) according to the manufacturer's instructions. Experiments were conducted 48h after siRNA transfection. Knockdown efficiency was confirmed by Western blotting.

\section{Antibodies, Reagents and Western blotting}

Western blot analysis was performed according to standard protocols. In all experiments, before the addition of EGF or erlotinib established cells were cultured overnight in serum free DMEM and primary GBM neurospheres were EGF starved overnight. Cells not treated with EGF or erlotinib were also serum or EGF starved. EGFR $(06-847,1 \mu \mathrm{g} / \mathrm{ml})$ antibody was from Millipore. pEGFR-1068 (2236), pERK (4376) ERK (4695), p34 (9251), JNK (9252), STAT3(9139), pAkt (0915) and pSTAT3 (9131) antibodies were from Cell Signaling Technology (Danvers, MA) were used a 1/1000 dilution; TNFR1 (sc-8436), ERK2 (sc-154). Akt (sc-1619), and $\beta$-Actin (sc-47778) were from Santa Cruz Biotechnology (Dallas, TX) were used at a concentration of $1 \mu \mathrm{g} / \mathrm{ml}$. GAS6 (AF885), pAxl (AF2228) and Axl (AF154) were from R\&D Biosystems (Minneapolis, $\mathrm{MN}$ ) were used at a concentration of $1 \mu \mathrm{g} / \mathrm{ml} \mathrm{c}$ JUN antibody (ab31419) was from Abcam (Cambridge, MA) and was used at $2 \mu \mathrm{g}$ per chromatin IP reaction.

Reagents: Recombinant human TNF and EGF were obtained from Peprotech (Rocky Hill, $\mathrm{NJ})$. Erlotinib and XL765 was purchased from SelleckChem (Houston, TX). Afatinib was bought from AstaTech, Inc. (Bristol, PA). Axl inhibitor R428, ERK inhibitor (U0126) and thalidomide were from Cayman Chemical (Ann Arbor, MI). Enbrel (Etanercept) was purchased from Mckesson Medical Supply (San Francisco CA). The JNK inhibitor SP600125, p38 Inhibitor SB203580, and NF- $\mathrm{kB}$ inhibitor BMS-345541 were obtained from EMD Millipore (Billerica, MA). Necrostatin-1 was obtained from Fisher Scientific (Pittsburg, PA). LPS was purchased from Sigma (St. Louis, MO). Cetuximab was provided by Imclone and used at a concentration of $10 \mu \mathrm{g} / \mathrm{ml}$ overnight.

\section{Chromatin Immunoprecipitation Assay}

GBM9 and U87EGFRwt cells were cultured in $15 \mathrm{~cm}$ plate per reaction for assay. ChIP assays were carried out using ChIP chromatin immunoprecipitation kit (Millipore) following manufacturer's instructions. The ChIP-enriched DNA samples were quantified by PCR using Platinum Taq DNA polymerase (Invitrogen). Putative AP-1 binding sites on GAS6 promoter were predicted by Using TFBIND program. The following primer pairs covering AP-1 sites were used: 5'-GGATCTGACCTCAGTGTATC-3' and 5'TGGTTGTCTTCACTAGCGAT-3'.

\section{cDNA Synthesis and Real Time PCR}

Total RNA was isolated by TRIzol Reagent (Ambion). cDNA Reverse Transcription were performed by using High-Capacity cDNA Reverse Transcription Kit (Applied Biosystems). PCR primers were synthesized by IDT (Coralville, IA). Each PCR was carried out in

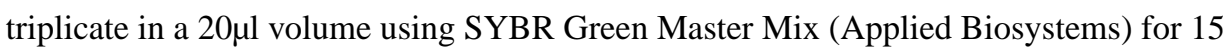


minutes at $95^{\circ} \mathrm{C}$ for initial denaturing, followed by 40 cycles of $95^{\circ} \mathrm{C}$ for $15 \mathrm{~s}$ and $60^{\circ} \mathrm{C}$ for $60 \mathrm{~s}$ in ViiA 7 Real-Time PCR System (Applied Biosystems). At least two independent experiments were done. Values for each gene were normalized to expression levels of $G A P D H$ mRNA. The following primers were used. TNF, $5^{\prime}-$ CCCAGGGACCTCTCTCTAATCA- $3^{\prime}$ and $5^{\prime}$-GCTACAGGCTTGTCACTCGG-3 ${ }^{\prime}$; GAS 6 , $5^{\prime}$-CATCAACAAGTATGGGTCTCCGT- ${ }^{\prime}$ and $5^{\prime}$ GTTCTCCTGGCTGCATTCGTTGA-3'; GAPDH, $5^{\prime}$ GTGAAGGTCGGAGTCAACGG-3' and 5' -TGATG-ACAAGCTTCCCGTTCTC-3'.

\section{ELISA}

$2 \times 10^{6}$ cells were incubated in serum-free or EGF free medium containing different concentrations of erlotinib or DMSO. After 48 hours, supernatant and cell lysates were collected. Supernatant medium was concentrated 5-10-fold with Pierce protein concentrator (ThermoFisher). TNF protein concentration in supernatant, cell lysates and tissue extracts was determined by ELISA using a commercial TNF detection kit (ThermoFisher) according to manufacturer's instructions. Additionally, GAS6 protein concentration in supernatant was measured by ELISA kit (LifeSpan BioSciences).

\section{Cell viability assay}

Cell viability assay was conducted using AlamarBlue cell viability assay kit (ThermoFisher) following manufacturer's protocol. Cells were cultured in Corning 96-well black plates with clear bottom (5000 cells/well). Drugs were added to cells for 72 hour following detection using POLARstar Omega Microplate Reader (excitation at 544nm and emission at 590nm) (BMG LABTECH, Germany).

\section{Animal studies}

4 to 6 weeks old female athymic nude mice were purchased from Charles River Laboratories. The mice were housed in plastic cages ( $4-5$ mice per cage) in a room with a 12 hour day-night cycle. $1 \times 10^{6}$ GBM9 cells were subcutaneously injected into the right flank of each nude mouse. After about 10 days post injection, all mice had developed subcutaneous tumors. The mice were randomly divided into control and treatment groups, mice were treated with drugs using the indicated doses. For combination treatment, both drugs were given concurrently for indicated periods. Tumor dimensions were measured by caliper every two days and tumor volumes calculated by the formula: volume $=$ length $\times$ width $\times$ width/2. Mice were sacrificed when tumors reached over $2000 \mathrm{~m}^{3}$ or 24 days from the first day of treatment.

For the orthotropic model, $1 \times 10^{5}$ GBM9 cells expressing firefly luciferase were injected into the right corpus striatum of the brains of 6-8-week-old nude mice using a stereotactic frame. When tumors became apparent on BLI (7 days after injection), mice were randomly divided into four groups (control gavage group, afatinib group, thalidomide group and afatinib plus thalidomide group, $\mathrm{n}=8$ ). The mice were treated with erlotinib $50 \mathrm{mg} / \mathrm{kg}$ by oral gavage and/or intraperitoneal (i.p.) injection of $150 \mathrm{mg} / \mathrm{kg}$ thalidomide for 10 consecutive days. Bioluminescence imaging (BLI) was performed to record the growth of tumor every 5 days. 
Kaplan-Maier survival curves were calculated using GraphPad Prism 7.0 software. Mice were monitored and sacrificed when neurological signs appeared or after 40 days.

To detect the in vivo levels of TNF, $1 \times 10^{6}$ GBM9 cells were injected into the right flanks of nude mice. When the subcutaneous tumor reached a volume of around $200 \mathrm{~mm}^{3}$, the mice were treated with erlotinib to monitor the upregulation of TNF protein. The animals were dosed consecutively for 1, 2, 7 or 14 days and then sacrificed. The animals without treatment were considered as control (0-day treatment). Tumor tissues were rinsed in PBS and protein was extracted to analyze the TNF levels using ELISA and also used for fwestern blot. To detect the protein expression in mice brain tumor tissues upon drug treatment, the same procedure was performed in orthotopic models. Additionally, with orthotopic models, mice were divided into four groups (control gavage group, erlotinib group, thalidomide group and erlotinib plus thalidomide, $\mathrm{n}=3$ ). After 48 hours treatment tumors were harvested and subjected to Western blot and immunostaining.

All animal studies were done under Institutional Animal Care and Use Committee-approved protocols.

\section{Immunohistochemistry}

Tumors from nude mice brains were fixed in $10 \%$ formalin and embedded in paraffin. Paraffin-embedded sections were cut at five-micrometer thickness. Immunohistochemical staining was performed using the $\mathrm{ABC}$ streptavidin-biotin method with the Vectastain $\mathrm{ABC}$ kit (Vector Laboratories, Burlingame, CA, USA) according to the manufacturer's protocol. Briefly, after deparaffinization and rehydration, endogenous peroxidase activity was quenched by a 10 min incubation in $3 \% \mathrm{H}_{2} \mathrm{O}_{2}$. For antigen retrieval, the tissue sections were boiled in $10 \mathrm{mM}$ sodium citrate buffer ( $\mathrm{pH}$ 6.0) for 20 minutes. Binding of primary antipERK (1:100, Cell Signaling Technology, 4376,) or anti-pJNK antibody (1:200, Cell signaling Technology, 4668, ) or anti-pAXL(1:400, R\&D, AF2228) was carried out overnight at $4^{\circ} \mathrm{C}$. The signal was detected by using the Sigmafast 3,3'-Diaminobenzidine tablets (DAB; Sigma, St. Louis MO). The sections were counterstained lightly with hematoxylin. The IHC staining intensity was scored semiquantitatively as: $0=$ No positive staining; $1=1-25 \%$ tumor cells stained, $2=26 \%-75 \%$ tumor cells stained and $3=>75 \%$ tumor cells stained.

\section{Cell death/Annexin assay}

Annexin assay was performed by using Annexin -V-FLUOS Staining kit (Roche applied Science). Cells $\left(1 \times 10^{6}\right)$ were plated in 6 well plates and treated with drugs or vehicle alone. After 72 hours cells were trypsinized and washed 2 times with $1 \times$ PBS. The cells were incubated for 15 minutes at room temperature with Propidium Iodide and Annexin -VFLUOS labeling solution in incubation buffer. Annexin and/or PI positive cells were detected by Flow Cytometry.

\section{Caspase-3/7 activity}

Caspase activity in the supernatant was measured using the Caspase-Glo 3/7 Assay (Promega, G8091) following manufacturer's instructions. Briefly, 72 hours after treatments, cells were washed by cold PBS and total cell lysates were prepared. $50 \mu \mathrm{g}$ of total protein 
per sample was added per well of a 96 well plate and was incubated with $100 \mu \mathrm{l}$ Caspase 3/7 reagent for 30 minutes. The luminescence of each sample was measured using a luminometer. All experiments included at least 3 replicates per group and were repeated 3 times.

\section{Statistical anaysis}

All data were analyzed for significance using GraphPad Prism 7.0 software. Error bars represent the means \pm s.e.m of three independent experiments if not indicated. We used 8 mice per group based on power analysis. This sample size calculation is based upon tumor volume measured at 4 weeks after drug administration. Specifications and assumptions for this calculation are: 1, a tumor volume change of $50 \%$ for the treated group as compared with the control group, 2, a standard deviation of $30 \%$ for tumor volume in each of the comparison groups, 3 , power of $85 \%$ and two-sided type I error rate of 5\%, 4, use of twosample t-test. (Total mice :32). Two-tailed unpaired Student's t-test were used for comparison of two data sets. The Mann-Whitney U test was applied to test the significant differences in IHC staining intensity between different groups. Data distribution was assumed to be normal, but this was not formally tested. Samples and animals were randomized for experimentation, data collection and analysis were not performed blind to the conditions of the experiments. At least 3 independent molecular and biochemical analyses were performed unless otherwise indicated. $P<0.05$ was considered statistically significant. * means that $P<0.05$, ** means that $P<0.01$, *** means that $<0.001$ and $* * * *$ indicates any $P$ value less than 0.0001 .

\section{Data availability}

The data supporting the findings of this study are available from the corresponding author upon reasonable request.

\section{Supplementary Material}

Refer to Web version on PubMed Central for supplementary material.

\section{Acknowledgments}

This work was supported in part by NIH grants RO1 NS062080 and by the Office of Medical Research, Departments of Veterans Affairs to AH(I01BX002559). SB is supported by grants from the National Institutes of Health (RO1CA149461, RO1CA197796 and R21CA202403) and the National Aeronautics and Space Administration (NNX16AD78G). This work was also supported by NIH grant 1R01CA194578 to DZ. We thank Drs. Frank Furnari and C. David. James, and Dr. Ezra Burstein for providing cell lines and reagents.

\section{References}

1. Sharma SV, Bell DW, Settleman J, Haber DA. Epidermal growth factor receptor mutations in lung cancer. Nat Rev Cancer. 2007; 7:169-181. [PubMed: 17318210]

2. Arteaga CL, Engelman JA. ERBB receptors: from oncogene discovery to basic science to mechanism-based cancer therapeutics. Cancer Cell. 2014; 25:282-303. [PubMed: 24651011]

3. Chong CR, Janne PA. The quest to overcome resistance to EGFR-targeted therapies in cancer. Nature medicine. 2013; 19:1389-1400.

4. Fan W, et al. MET-independent lung cancer cells evading EGFR kinase inhibitors are therapeutically susceptible to BH3 mimetic agents. Cancer Res. 2011; 71:4494-4505. [PubMed: 21555370] 
5. Lee HJ, et al. Drug resistance via feedback activation of Stat3 in oncogene-addicted cancer cells. Cancer Cell. 2014; 26:207-221. [PubMed: 25065853]

6. Verhaak RG, et al. Integrated genomic analysis identifies clinically relevant subtypes of glioblastoma characterized by abnormalities in PDGFRA, IDH1, EGFR, and NF1. Cancer Cell. 2010; 17:98-110. [PubMed: 20129251]

7. Hatanpaa KJ, Burma S, Zhao D, Habib AA. Epidermal growth factor receptor (EGFR) in glioma: Signal transduction, neuropathology, imaging and radioresistance. Neoplasia. 2010; 12:675-684. [PubMed: 20824044]

8. Mellinghoff IK, et al. Molecular determinants of the response of glioblastomas to EGFR kinase inhibitors. N Engl J Med. 2005; 353:2012-2024. [PubMed: 16282176]

9. Karpel-Massler G, Schmidt U, Unterberg A, Halatsch ME. Therapeutic inhibition of the epidermal growth factor receptor in high-grade gliomas: where do we stand? Mol Cancer Res. 2009; 7:10001012. [PubMed: 19584260]

10. Akhavan D, et al. De-repression of PDGFRbeta transcription promotes acquired resistance to EGFR tyrosine kinase inhibitors in glioblastoma patients. Cancer Discov. 2013; 3:534-547. [PubMed: 23533263]

11. Nathanson DA, et al. Targeted therapy resistance mediated by dynamic regulation of extrachromosomal mutant EGFR DNA. Science. 2014; 343:72-76. [PubMed: 24310612]

12. Barkovich KJ, et al. Kinetics of inhibitor cycling underlie therapeutic disparities between EGFRdriven lung and brain cancers. Cancer Discov. 2012; 2:450-457. [PubMed: 22588882]

13. Wykosky J, et al. A urokinase receptor-Bim signaling axis emerges during EGFR inhibitor resistance in mutant EGFR glioblastoma. Cancer Res. 2015; 75:394-404. [PubMed: 25432173]

14. Chakraborty $\mathrm{S}$, et al. Constitutive and ligand-induced EGFR signalling triggers distinct and mutually exclusive downstream signalling networks. Nat Commun. 2014; 5:5811. [PubMed: 25503978]

15. Guo G, et al. Ligand-Independent EGFR Signaling. Cancer Res. 2015; 75:3436-3441. [PubMed: 26282175]

16. Acquaviva J, et al. Chronic activation of wild-type epidermal growth factor receptor and loss of Cdkn2a cause mouse glioblastoma formation. Cancer Res. 2011; 71:7198-7206. [PubMed: 21987724]

17. Endres NF, et al. Conformational coupling across the plasma membrane in activation of the EGF receptor. Cell. 2013; 152:543-556. [PubMed: 23374349]

18. Ramnarain DB, et al. Differential gene expression analysis reveals generation of an autocrine loop by a mutant epidermal growth factor receptor in glioma cells. Cancer Res. 2006; 66:867-874. [PubMed: 16424019]

19. Wong AJ, et al. Structural alterations of the epidermal growth factor receptor gene in human gliomas. Proc Natl Acad Sci U S A. 1992; 89:2965-2969. [PubMed: 1557402]

20. Gil Del Alcazar CR, Todorova PK, Habib AA, Mukherjee B, Burma S. Augmented HR Repair Mediates Acquired Temozolomide Resistance in Glioblastoma. Mol Cancer Res. 2016; 14:928940. [PubMed: 27358111]

21. Pingle SC, et al. In silico modeling predicts drug sensitivity of patient-derived cancer cells. J Transl Med. 2014; 12:128. [PubMed: 24884660]

22. Puliyappadamba VT, et al. Opposing Effect of EGFRWT on EGFRvIII-Mediated NF-kappaB Activation with RIP1 as a Cell Death Switch. Cell Rep. 2013; 4:764-775. [PubMed: 23972990]

23. Sarkaria JN, et al. Use of an orthotopic xenograft model for assessing the effect of epidermal growth factor receptor amplification on glioblastoma radiation response. Clin Cancer Res. 2006; 12:2264-2271. [PubMed: 16609043]

24. Stommel JM, et al. Coactivation of receptor tyrosine kinases affects the response of tumor cells to targeted therapies. Science. 2007; 318:287-290. [PubMed: 17872411]

25. Huang PH, et al. Quantitative analysis of EGFRvIII cellular signaling networks reveals a combinatorial therapeutic strategy for glioblastoma. Proc Natl Acad Sci U S A. 2007; 104:1286712872. [PubMed: 17646646] 
26. Pillay V, et al. The plasticity of oncogene addiction: implications for targeted therapies directed to receptor tyrosine kinases. Neoplasia. 2009; 11:448-458. 442 p following 458. [PubMed: 19412429]

27. Greenall SA, et al. EGFRvIII-mediated transactivation of receptor tyrosine kinases in glioma: mechanism and therapeutic implications. Oncogene. 2015; 34:5277-5287. [PubMed: 25659577]

28. Awad AJ, Burns TC, Zhang Y, Abounader R. Targeting MET for glioma therapy. Neurosurgical focus. 2014; 37:E10.

29. Zhu Y, Shah K. Multiple lesions in receptor tyrosine kinase pathway determine glioblastoma response to pan-ERBB inhibitor PF-00299804 and PI3K/mTOR dual inhibitor PF-05212384. Cancer Biol Ther. 2014; 15:815-822. [PubMed: 24658109]

30. Blakely CM, et al. NF-kappaB-activating complex engaged in response to EGFR oncogene inhibition drives tumor cell survival and residual disease in lung cancer. Cell Rep. 2015; 11:98110. [PubMed: 25843712]

31. Wajant H, Pfizenmaier K, Scheurich P. Tumor necrosis factor signaling. Cell Death Differ. 2003; 10:45-65. [PubMed: 12655295]

32. Higuchi M, Aggarwal BB. TNF induces internalization of the p60 receptor and shedding of the p 80 receptor. J Immunol. 1994; 152:3550-3558. [PubMed: 8144934]

33. Deng L, Ding W, Granstein RD. Thalidomide inhibits tumor necrosis factor-alpha production and antigen presentation by Langerhans cells. The Journal of investigative dermatology. 2003; 121:1060-1065. [PubMed: 14708607]

34. Gross ND, et al. Inhibition of Jun NH2-terminal kinases suppresses the growth of experimental head and neck squamous cell carcinoma. Clin Cancer Res. 2007; 13:5910-5917. [PubMed: 17908987]

35. Groves MD, et al. A North American brain tumor consortium (NABTC 99-04) phase II trial of temozolomide plus thalidomide for recurrent glioblastoma multiforme. J Neurooncol. 2007; 81:271-277. [PubMed: 17031561]

36. $\mathrm{Gu} \mathrm{X}$, et al. Intraperitoneal injection of thalidomide attenuates bone cancer pain and decreases spinal tumor necrosis factor-alpha expression in a mouse model. Molecular pain. 2010; 6:64. [PubMed: 20923560]

37. Prahallad A, et al. Unresponsiveness of colon cancer to BRAF(V600E) inhibition through feedback activation of EGFR. Nature. 2012; 483:100-103. [PubMed: 22281684]

38. Duncan JS, et al. Dynamic reprogramming of the kinome in response to targeted MEK inhibition in triple-negative breast cancer. Cell. 2012; 149:307-321. [PubMed: 22500798]

39. Huang PH, Xu AM, White FM. Oncogenic EGFR signaling networks in glioma. Sci Signal. 2009; 2:re6. [PubMed: 19738203]

40. Li L, et al. An EGFR wild type-EGFRvIII-HB-EGF feed-forward loop regulates the activation of EGFRvIII. Oncogene. 2014; 33:4253-4264. [PubMed: 24077285]

41. Fan QW, et al. EGFR phosphorylates tumor-derived EGFRvIII driving STAT3/5 and progression in glioblastoma. Cancer Cell. 2013; 24:438-449. [PubMed: 24135280]

42. Jahani-Asl A, et al. Control of glioblastoma tumorigenesis by feed-forward cytokine signaling. Nat Neurosci. 2016; 19:798-806. [PubMed: 27110918]

43. de la Iglesia N, et al. Identification of a PTEN-regulated STAT3 brain tumor suppressor pathway. Genes Dev. 2008; 22:449-462. [PubMed: 18258752]

44. Rich JN, et al. Phase II trial of gefitinib in recurrent glioblastoma. J Clin Oncol. 2004; 22:133-142. [PubMed: 14638850]

45. Brown PD, et al. Phase I/II trial of erlotinib and temozolomide with radiation therapy in the treatment of newly diagnosed glioblastoma multiforme: North Central Cancer Treatment Group Study N0177. J Clin Oncol. 2008; 26:5603-5609. [PubMed: 18955445]

46. Peereboom DM, et al. Phase II trial of erlotinib with temozolomide and radiation in patients with newly diagnosed glioblastoma multiforme. J Neurooncol. 2010; 98:93-99. [PubMed: 19960228]

47. Schuster J, et al. A phase II, multicenter trial of rindopepimut (CDX-110) in newly diagnosed glioblastoma: the ACT III study. Neuro Oncol. 2015; 17:854-861. [PubMed: 25586468] 
48. Inda MD, et al. Tumor heterogeneity is an active process maintained by a mutant EGFR-induced cytokine circuit in glioblastoma. Genes Dev. 2010; 24:1731-1745. [PubMed: 20713517]

49. Furnari FB, Cloughesy TF, Cavenee WK, Mischel PS. Heterogeneity of epidermal growth factor receptor signalling networks in glioblastoma. Nat Rev Cancer. 2015; 15:302-310. [PubMed: 25855404]

50. Stupp R, et al. Radiotherapy plus concomitant and adjuvant temozolomide for glioblastoma. N Engl J Med. 2005; 352:987-996. [PubMed: 15758009] 


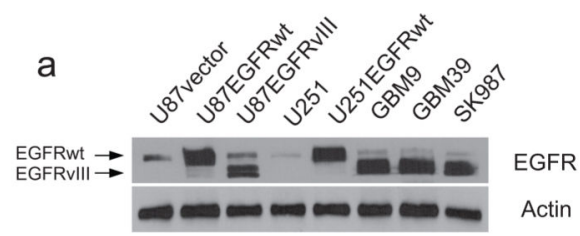

b

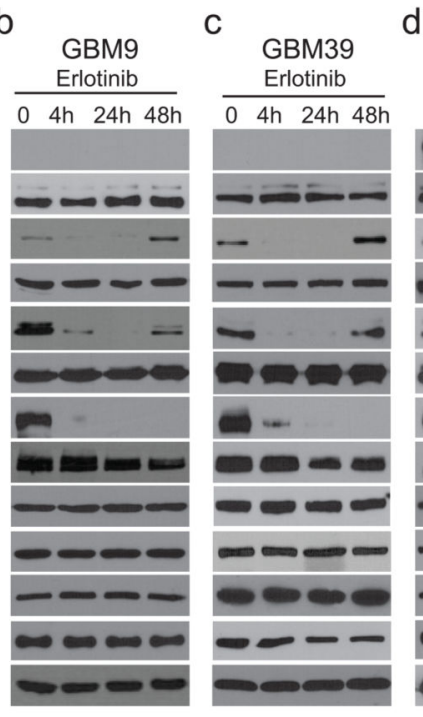

d

SK987
0 Eh $24 \mathrm{~h} 48 \mathrm{~h}$
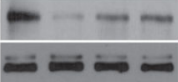

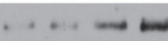
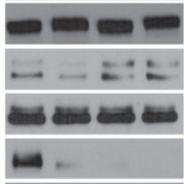

=

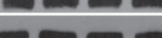

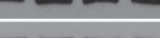

$-\infty-$
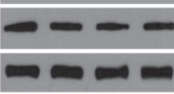

ex

e U87EGFRwt $f$ U87EGFRvIII $\frac{\text { Erlotinib }}{04 h 24 h \text { Erh }} \frac{\text { Erlotinib }}{0 \text { 4h } 24 \mathrm{~h} 48 \mathrm{~h}}$

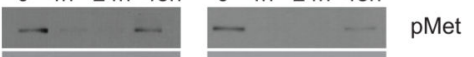

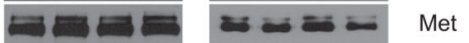

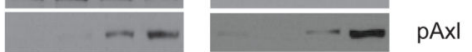

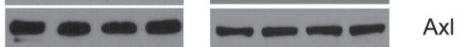

$=-\quad-\quad-\mathrm{pERK}$

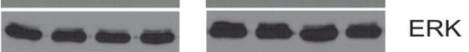

- 2 pEGFR

- $\rightarrow$ EGFR

ए

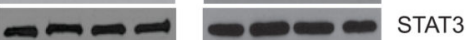

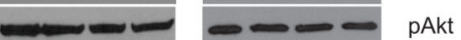

- - - Akt

$\infty-\infty$

$--$

Akt

Actin

g

h GBM39

i

U87EGFRwt ${ }^{j}$

jus7Egrnvil

Erlotinib 48h
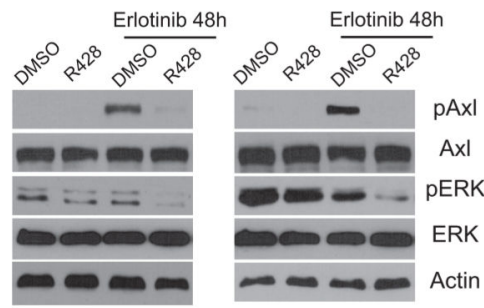

$\mathrm{k}$

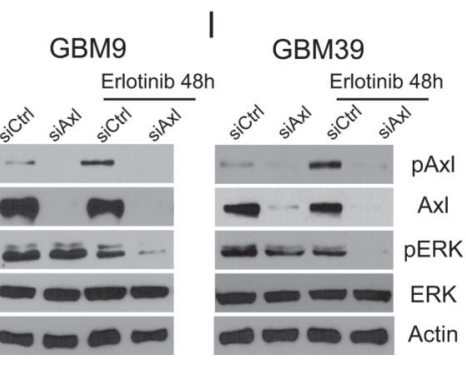

$m$ U87EGFRwt

$n$ U87EGFRvIII

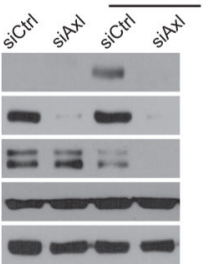

Erlotinib 48h

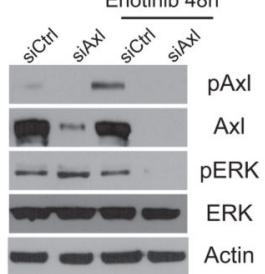

Figure 1. EGFR inhibition triggers an adaptive response in glioma cells

(a) Western blot showing EGFR levels in established GBM cell lines and patient derived primary GBM neurospheres. (b) Patient derived primary GBM neurospheres (GBM9) were exposure to erlotinib $(1 \mu \mathrm{M})$ for the indicated times followed by Western blot with the indicated antibodies. (c-d) A similar experiment in patient derived GBM neurospheres from two different patients (GBM39 and SK987). (e) U87EGFR cells were treated with erlotinib $(1 \mu \mathrm{M})$ for the indicated times followed by Western blot with the indicated antibodies. (f) Similar experiment was conducted in U87EGFRvIII cells. (g-j) Axl was inhibited using 
R428 (1 $\mu \mathrm{M})$, a specific inhibitor of Axl. Cells were exposed to Erlotinib followed by Western blot. Erlotinib-induced ERK activation is inhibited when the Axl inhibitor is used in both established GBM cell lines as well as patient derived neurospheres. $(\mathrm{k}-\mathrm{n})$ siRNA knockdown of Axl results in an inhibition of erlotinib induced ERK activation in both established cell lines as well as patient derived neurospheres. Control siRNA or Axl siRNA was transfected into cells (for 48h), followed by addition of erlotinib for 48h and WB with indicated antibodies. Western blots shown in a-n are representative of at least three independent replicates. Full-length blots are presented in Supplementary Figure 11. 

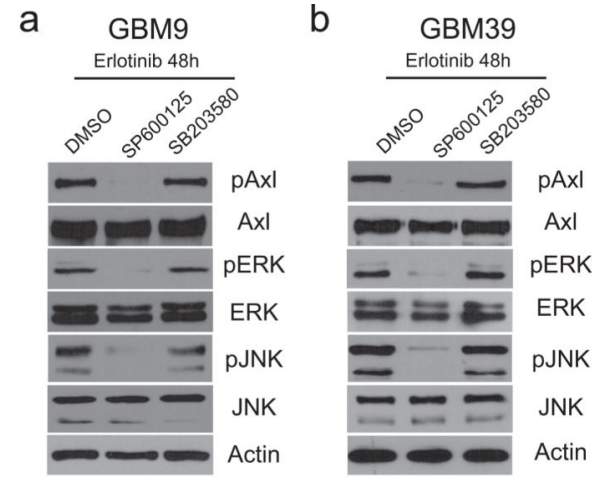

d

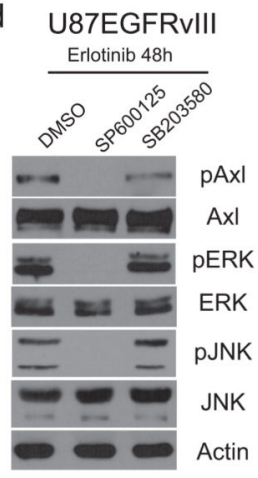

j g

U87EGFRwt

Erlotinib 48h

siCtrl siJNK

-. pAxl

- AxI

- pERK

$=$ ERK

- pJNK

- JNK

$=$ Actin h

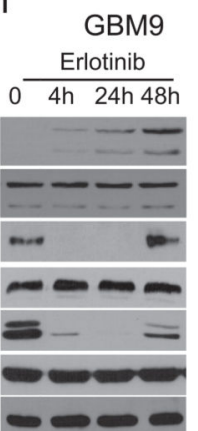

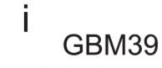

$0 \frac{\text { Erlotinib }}{4 \mathrm{~h} \mathrm{24h} \mathrm{48h}}$

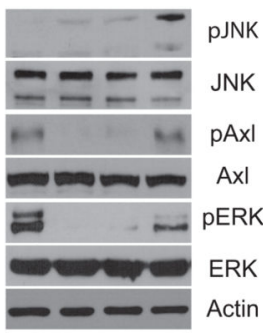

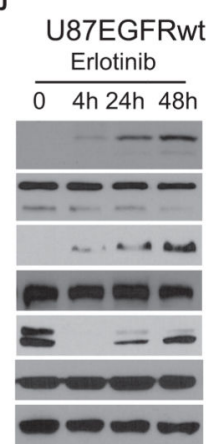

$\mathrm{k}$

U87EGFRvIII

Erlotinib

0 4h 24h 48h

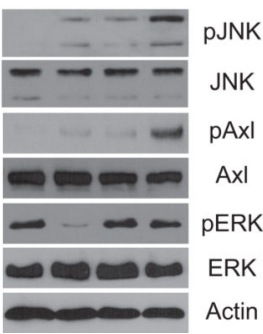

e

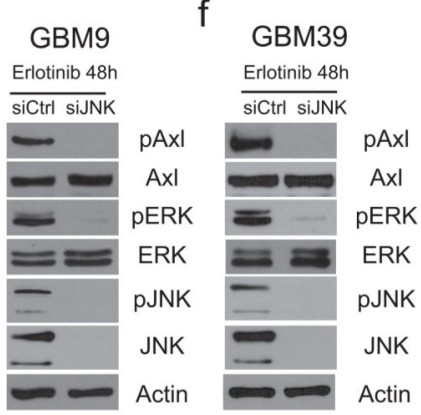

Figure 2. EGFR inhibition induced Axl and ERK activation is mediated by JNK

(a-b) Patient derived primary GBM neurospheres were exposed to erlotinib for 48 hours in the presence or absence of JNK inhibitor SP600125 $(1 \mu \mathrm{M})$ or p38 inhibitor SB203580 $(10 \mu \mathrm{M})$ followed by Western blot with the indicated antibodies. (c-d) U87EGFRwt or U87EGFRvIII cells were exposed to erlotinib for 48 hours in the presence or absence of SP600125 or SB203580 followed by Western blot with the indicated antibodies. (e-f) siRNA knockdown for JNK1 and JNK2 was conducted in GBM9 and GBM39 neurospheres followed by exposure to erlotinib for $48 \mathrm{~h}$ and Western blot with the indicated antibodies. $(\mathrm{g})$ A similar experiment was done in U87EGFRwt cells. ( $\mathrm{h}-\mathrm{k}$ ) shows that JNK is activated in response to erlotinib in patient derived primary neurospheres as well as established GBM cell lines as determined by the phosphorylation of JNK. Western blots shown in a-k are representative of at least three independent replicates. Full-length blots are presented in Supplementary Figure 12. (1) A luciferase reporter assay shows that EGFR inhibition with erlotinib results in an increase in AP-1 transcriptional activity in GBM9 and U87EGFRwt cells. Erlotinib was used for $24 \mathrm{~h}(1 \mu \mathrm{M})$. GBM9: Ctrl vs erlotinib: $P=0.0056, \mathrm{t}=5.43$, d.f. $=4$ $* * P<0.01$; U87EGFRwt: Ctrl vs erlotinib: $P=0.0061, \mathrm{t}=5.31$, d.f.=4. $* * P<0.01$. Data are presented as mean \pm s.e.m. Significant difference analyzed by an unpaired Student's t-test ( $n$ $=3$ biologically independent experimental replicates). 

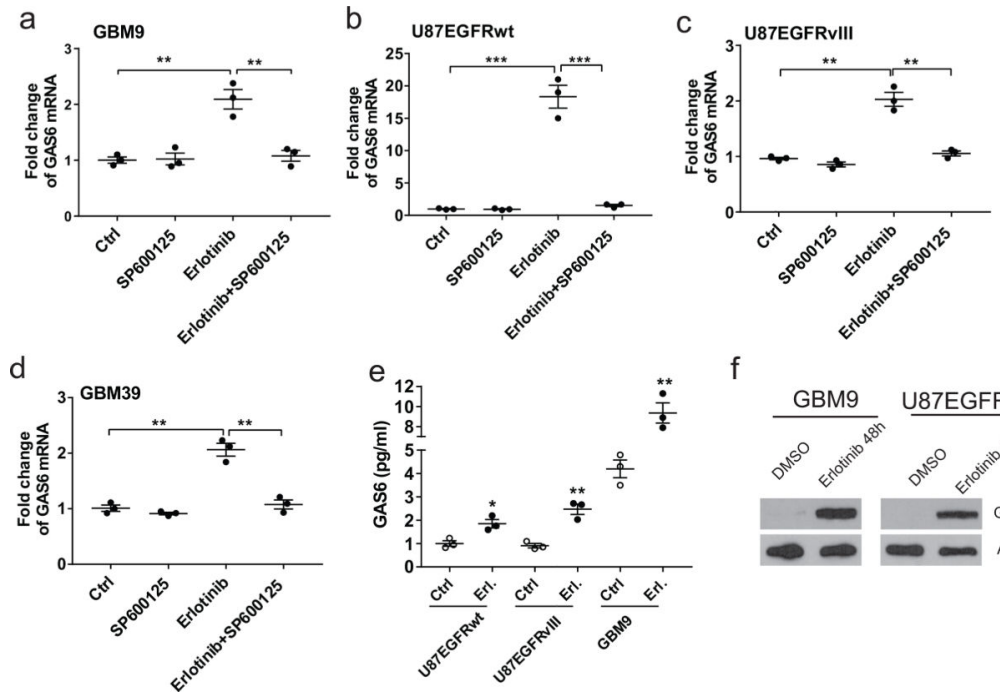

g

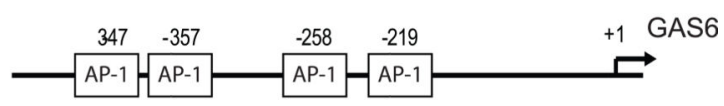

$\mathrm{h}$

i
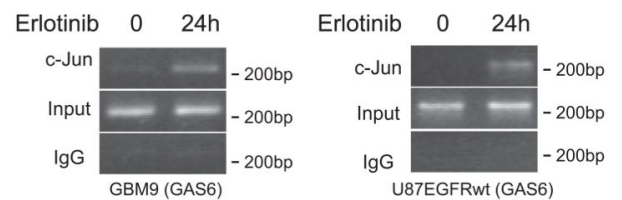

Figure 3. EGFR inhibition leads to increased GAS6 levels via a JNK dependent mechanism (a) GBM9 neurospheres were exposed to erlotinib in the absence or presence of JNK inhibitor SP600125 for $24 \mathrm{~h}(1 \mu \mathrm{M})$ for $24 \mathrm{~h}$ followed by quantitative real time PCR for GAS6 mRNA. GAS6 is increased upon EGFR inhibition, and this increase is blocked by JNK inhibition. Ctrl vs erlotinib: $P=0.0039$, t=5.98, d.f.=4; erlotinib vs erlotinib+SP600125: $P$ $=0.0070, \mathrm{t}=5.10$, d.f.=4. $(\mathrm{b}-\mathrm{d})$ A similar experiment was undertaken in U87EGFRwt, U87EGFRvIII and patient derived neurosphere GBM39. (b) Ctrl vs erlotinib: $P=0.0006$, $\mathrm{t}=9.83$, d.f. $=4$; erlotinib vs erlotinib+SP600125: $P=0.0007$, $\mathrm{t}=9.48$, d.f.=4. (c) Ctrl vs erlotinib: $P=0.0011, \mathrm{t}=8.39$, d.f.=4; erlotinib vs erlotinib+SP600125: $P=0.0018, \mathrm{t}=7.32$, d.f.=4. (d) Ctrl vs erlotinib: $P=0.0012$, t=8.20, d.f.=4; erlotinib vs erlotinib+SP600125: $P$ $=0.0022, \mathrm{t}=6.97$, d.f.=4. (e) An ELISA showing the erlotinib $(1 \mu \mathrm{M}$ for $24 \mathrm{~h})$ induced increase in GAS6 levels at a protein level. U87EGFRwt: Ctrl vs erlotinib: $P=0.0175$, $\mathrm{t}=3.90$, d.f. $=4$; U87EGFRvIII: Ctrl vs erlotinib: $P=0.0030, \mathrm{t}=6.45$, d.f.=4; GBM9: Ctrl vs erlotinib: $P$ $=0.0087, \mathrm{t}=4.80$, d.f. $=4$. Data are presented as mean \pm s.e.m; $* P<0.05$, $* * P<0.01, * * * P<$ 0.001 from two-tailed unpaired Student's t-test $(n=3$ biologically independent experimental replicates). (f) Western blot showing increase of GAS6 protein in both GBM9 and U87EGFRwt cells upon erlotinib treatment Western blots shown in a-k are representative of at least three independent replicates. Full-length blots are presented in Supplementary Figure 12. (g) A schematic of the GAS6 promoter showing AP-1 sites. (h-i) ChIP assay showing the presence of c-Jun on the $G A S 6$ promoter in response to erlotinib ( $1 \mu \mathrm{M}$ for $24 \mathrm{~h})$ in GBM9 neurospheres and in U87EGFRwt cells. ChIP results are representative of at least three independent replicates. Full-length DNA agarose gels are presented in Supplementary in Figure 12. 

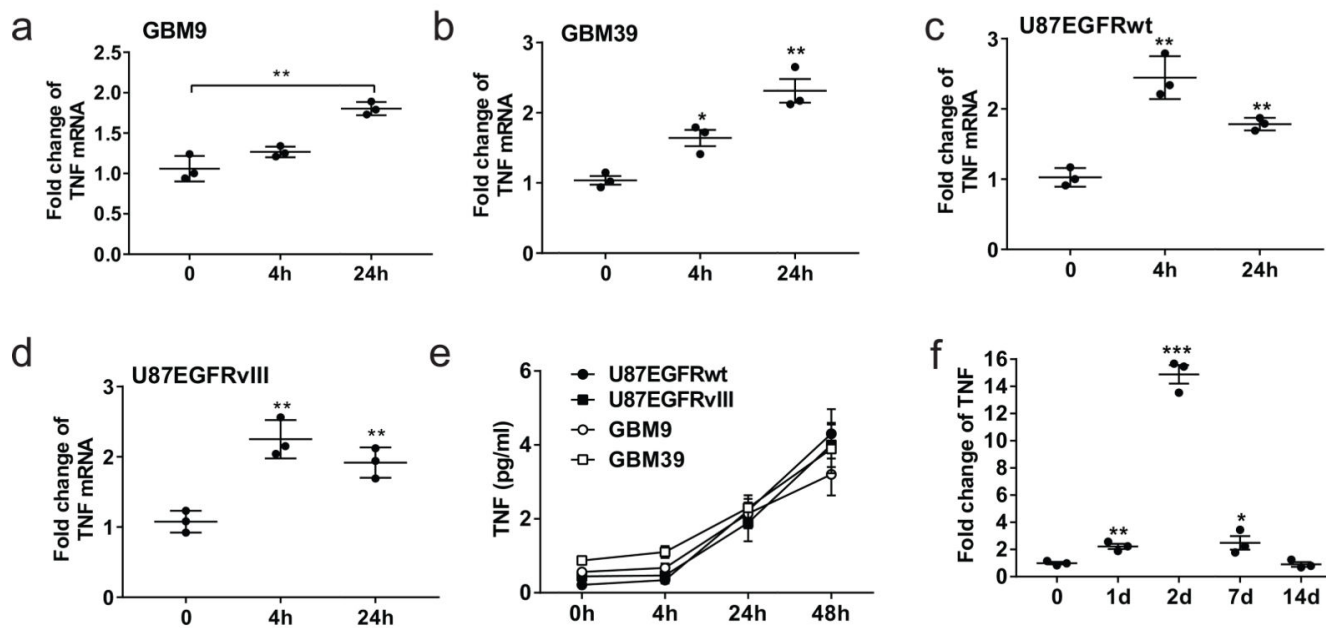

e

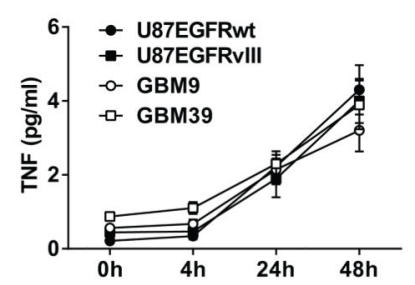

h

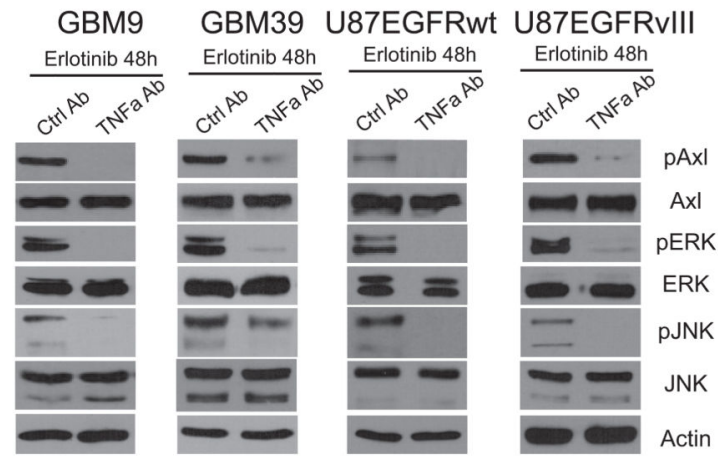

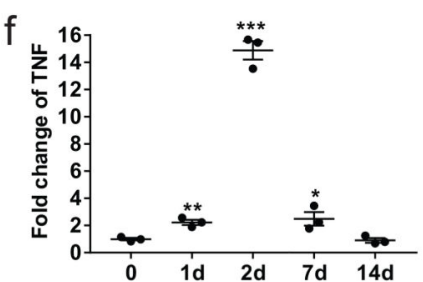

9 Subcutanous Tumor

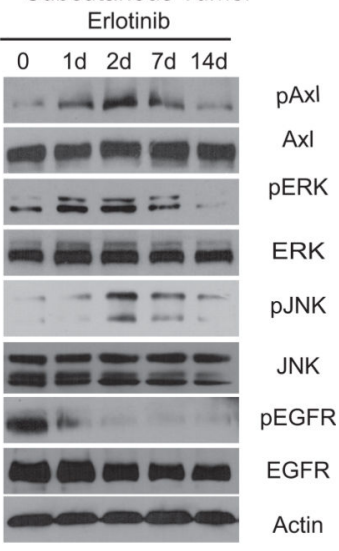

Figure 4. EGFR inhibition leads to increased TNF signaling that triggers an adaptive signaling pathway

(a-b) EGFR inhibition leads to an increase in TNFmRNA in patient derived GBM9 and GBM39 neurospheres. Cells were exposed to erlotinib $(100 \mathrm{nM})$ for the times indicated followed by qRT-PCR for $T N F$ mRNA. (a) 0 vs $24 \mathrm{~h}: P=0.0019, \mathrm{t}=7.22$, d.f. $=4$. (b) 0 vs $4 \mathrm{~h}$ : $P=0.0102$, $\mathrm{t}=4.58$, d.f. $=4$; 0 vs $24 \mathrm{~h}: P=0.0021$, $\mathrm{t}=7.10$, d.f. $=4$. $(\mathrm{c}-\mathrm{d})$ A similar experiment was conducted in U87EGFRwt and U87EGFRvIII cells using an erlotinib concentration of 1 $\mu$ M. (c) 0 vs 4h: $P=0.0018$, t=7.41, d.f. $=4$; 0 vs $24 \mathrm{~h}: P=0.0012$, t=8.20, d.f.=4. (d) 0 vs 4 h: $P=0.0030, \mathrm{t}=6.46$, d.f.=4; 0 vs $24 \mathrm{~h}: P=0.0054$, $\mathrm{t}=5.47$, d.f.=4. (e) A TNF ELISA was performed on supernatants from erlotinib treated U87EGFRwt and U87EGFRvIII cells $(1 \mu \mathrm{M})$ and GBM9 and GBM39 neurospheres (100 nM). U87EGFRwt: 0 vs 24h: $P=0.0056$, $\mathrm{t}=5.42$, d.f. $=4$; 0 vs 48h: $P=0.0006$, $\mathrm{t}=10.4$, d.f.=4; U87EGFRVIII: 0 vs $24 \mathrm{~h}: P=0.0022$, $\mathrm{t}=6.98$, d.f.=4; 0 vs 48h: $P=0.0083$, t=4.86, d.f.=4; GBM9: 0 vs $24 \mathrm{~h}: P=0.01$, $\mathrm{t}=4.6$, d.f. $=4$; 0 vs 48h: $P=0.0043$, t=5.84, d.f.=4; GBM39: 0 vs 24 h: $P=0.0189$, $\mathrm{t}=3.82$, d.f.=4; 0 h vs 48 h: $P=0.0024, \mathrm{t}=6.81$, d.f.=4. Data are presented as mean \pm s.e.m; $* P \leq 0.05$, ** $P \leq 0.01$, *** $P \leq$ 0.001 from two-tailed unpaired Student's t-test $(\mathrm{n}=3$ biologically independent experimental replicates). (f) Time course of TNF upregulation in mouse tumors exposed to erlotinib $50 \mathrm{mg} / \mathrm{kg}$ for the indicated time points after formation of subcutaneous tumors $(\mathrm{n}=3)$. Tumors were removed following erlotinib exposure followed by TNF ELISA on protein extracts. 0 vs $1 \mathrm{~d}: P=0.0045$, t=5.77, d.f. $=4 ; 0$ vs $2 \mathrm{~d}: P=0.0002$, t=13.92, d.f. $=4 ; 0$ vs $7 \mathrm{~d}: P=0.0245$, 
$\mathrm{t}=3.52$, d.f. $=4$. Data are presented as mean \pm s.e. $\mathrm{m} ; * P<0.05, * * P<0.01, * * * P<0.001$ from a two-tailed unpaired Student's t-test. (g) Shows signal transduction in tumors exposed to erlotinib $(50 \mathrm{mg} / \mathrm{kg}$ ) for the indicated time points. (h) A neutralizing antibody to TNF $(1 \mu \mathrm{g} / \mathrm{ml})$ blocked erlotinib induced activation of Axl, ERK and JNK in GBM9 and GBM39 neurospheres and U87EGFRwt and U87EGFRvIII cell lines, while a control antibody had no effect. (i) siRNA knockdown of TNFR1 blocked erlotinib induced activation of Axl, ERK and JNK in GBM9 and GBM39 neurospheres and also U87EGFRwt and U87EGFRvIII cell lines, while control (scrambled siRNA) had no effect. Western blots shown in g-I are representative of at least three independent replicates. Full-length blots are presented in Supplementary Figure 13. 
a

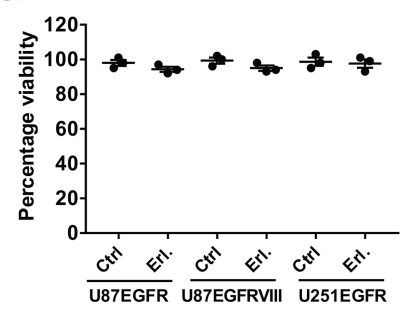

b

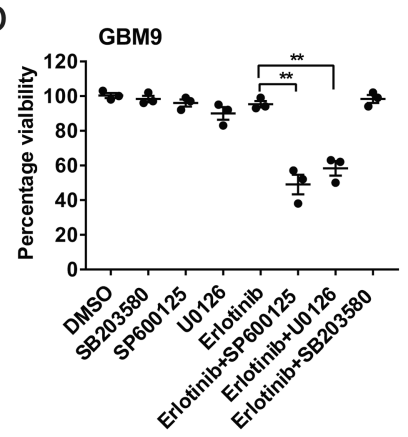

d

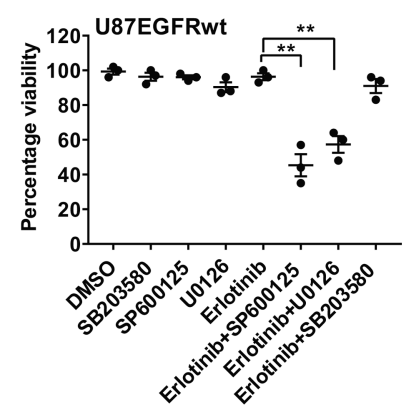

e

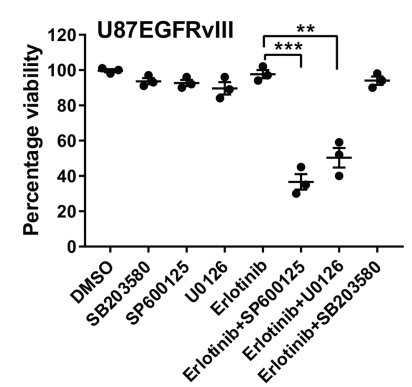

C

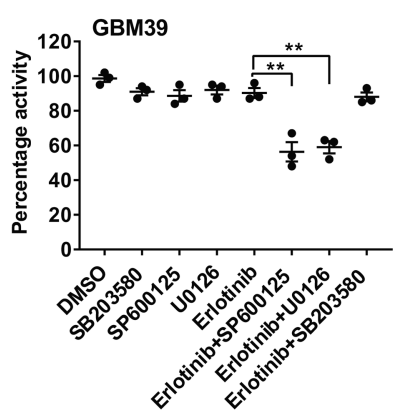

f

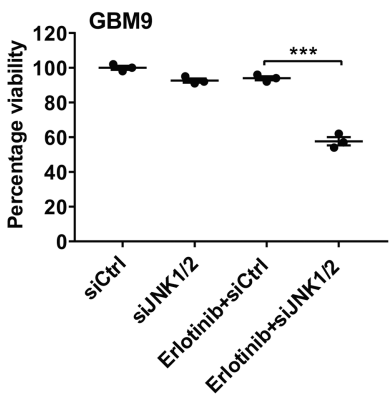

h

9

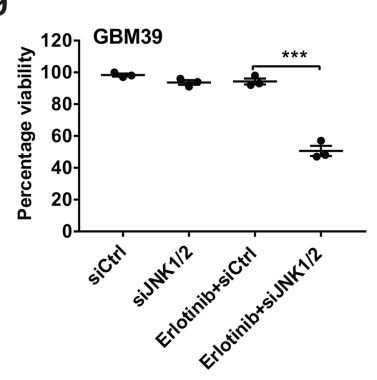

k

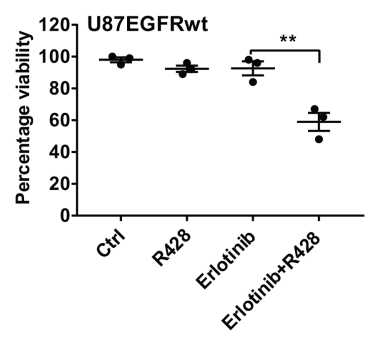

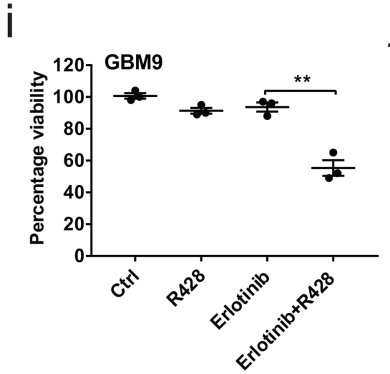

m
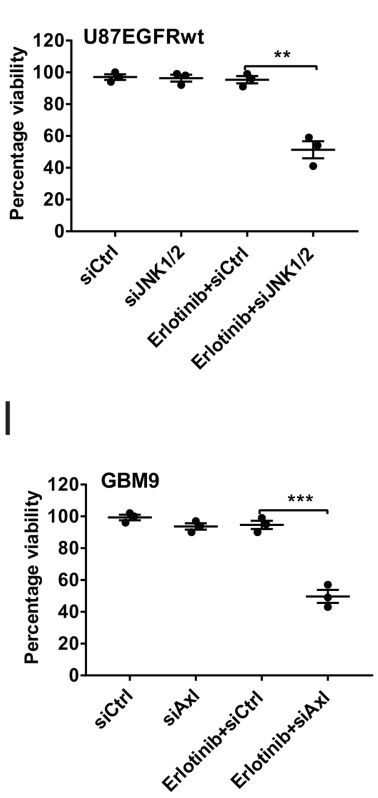

I

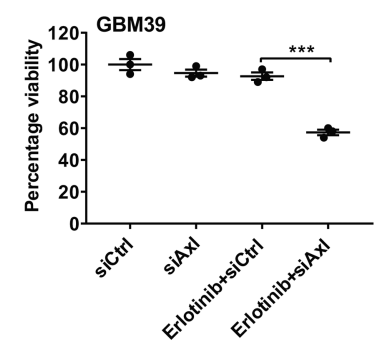

j

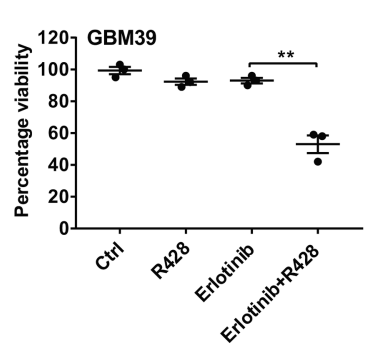

$\mathrm{n}$

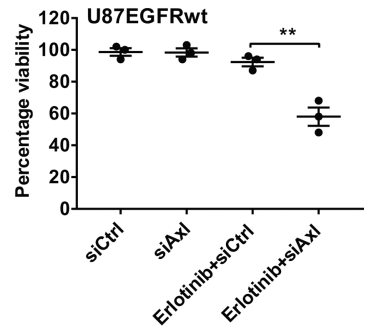

Figure 5. Inhibition of JNK and ERK renders glioma cells sensitive to EGFR inhibition

(a) AlamarBlue assay in established GBM cell lines exposed to erlotinib $(10 \mu \mathrm{M})$. Cells are completely resistant to the effects of EGFR inhibition. (b-c) Patient derived GBM9 or GBM39 neurospheres were exposed to erlotinib (100nM) with or without JNK inhibitor SP600125 $(1 \mu \mathrm{M})$, p38 inhibitor SB203580 $(10 \mu \mathrm{M})$, or ERK inhibitor U0126 $(1 \mu \mathrm{M})$, followed by Alamarblue Cell Survival Assay after $72 \mathrm{~h}$ of inhibitor exposure. (b) Erlotinib vs erlotinib+SP600125: $P=0.0015, \mathrm{t}=7.75$, d.f.=4; erlotinib vs erlotinib+U0126: $P=0.0013$, $\mathrm{t}=8.10$, d.f.=4. (c) erlotinib vs erlotinib+SP600125: $P=0.0057, \mathrm{t}=5.41$, d.f.=4; Erlotinib vs 
Erlotinib+U0126: $P=0.0023, \mathrm{t}=6.93$, d.f.=4. $(\mathrm{d}-\mathrm{e})$ A similar experiment was conducted in U87EGFRwt and U87EGFRvIII cells. (d) erlotinib vs erlotinib+SP600125: $P$ $=0.0016, \mathrm{t}=7.61$, d.f. $=4$; Erlotinib vs Erlotinib+U0126: $P=0.0017, \mathrm{t}=7.48$, d.f. $=4$. (e) erlotinib vs erlotinib+SP600125: $P=0.0003, \mathrm{t}=12.23$, d.f. $=4$; erlotinib vs erlotinib+U0126: $P=0.0014, \mathrm{t}=7.87$, d.f. $=4$. (f) siRNA knockdown of JNK1 and JNK2 in GBM9 neurospheres results in an enhanced sensitivity to erlotinib, whereas control siRNA has no effect. Erlotinib + siCtrl vs erlotinib+siJNK1/2: $P=0.0002$, $\mathrm{t}=13.96$, d.f. $=4$. $(\mathrm{g})$ siRNA knockdown of JNK1 and JNK2 in GBM39 neurosphere cells has a similar effect. Erlotinib+siCtrl vs Erlotinib + siJNK1/2: $P=0.0003, \mathrm{t}=11.86$, d.f.=4. (h) U87EGFRwt cells results in an enhanced sensitivity to erlotinib, whereas control siRNA has no effect. Erlotinib+siCtrl vs erlotinib + siJNK1/2: $P=0.0017, \mathrm{t}=7.52$, d.f.=4. (i-j) Patient derived GBM9 or GBM39 neurospheres were exposed to erlotinib $(100 \mathrm{nM})$ with or without Axl inhibitor R428 $(1 \mu \mathrm{M})$ followed by Alamarblue Cell Survival Assay after 72h. (i) Erlotinib vs erlotinib+R428: $P$ $=0.0025, \mathrm{t}=6.75$, d.f. $=4$. (i) Erlotinib vs erlotinib+R428: $P=0.0023, \mathrm{t}=6.93$, d.f. $=4$. (k) A similar experiment was done in U87EGFRwt cells using an erlotinib concentration of $1 \mu \mathrm{M}$. Erlotinib vs erlotinib+R428: $P=0.0094, \mathrm{t}=4.69$, d.f. $=4$. $(1-\mathrm{n})$ siRNA knockdown of $\mathrm{Axl}$ in GBM9 and GBM39 neurospheres or U87EGFRwt cells sensitizes cells to the effect of erlotinib but not control siRNA as determined by Alamarblue Cell Viability Assay. (1) Erlotinib+siCtrl vs erlotinib+siAxl: $P=0.0004, \mathrm{t}=11.23$, d.f. $=4$. (m) Erlotinib+siCtrl vs erlotinib+siAxl: $P=0.0003, \mathrm{t}=12.80$, d.f. $=4$. (n) Erlotinib+siCtrl vs erlotinib+R428: $P$ $=0.0058, \mathrm{t}=5.38$, d.f. $=4$. Data are presented as mean \pm s.e. $m ; * * P<0.01, * * * P<0.001$ from two-tailed unpaired Student's t-test ( $\mathrm{n}=3$ biologically independent experimental replicates). 
a
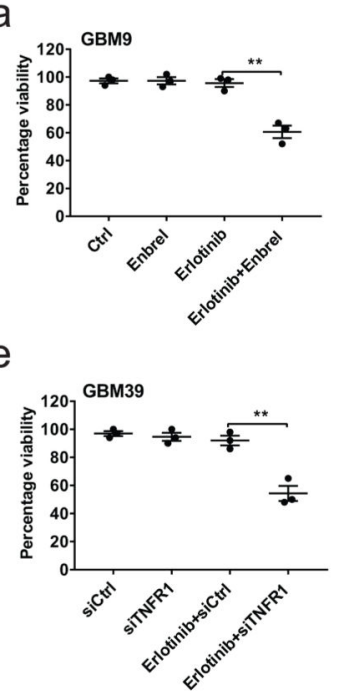

i

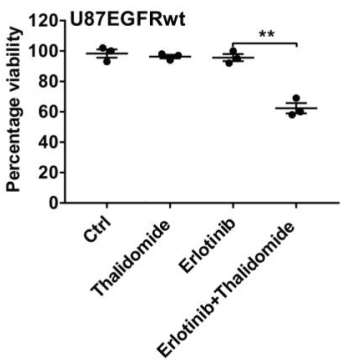

I

U87EGFRwt U87EGFRwt

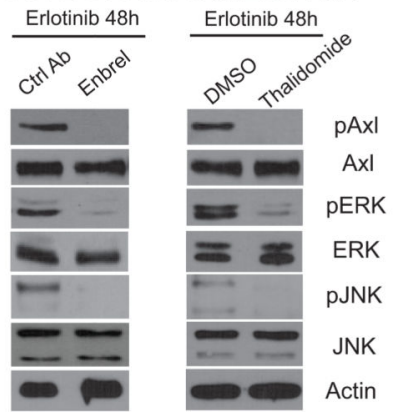

b

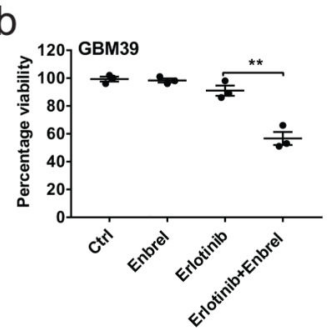

$\mathrm{f}$

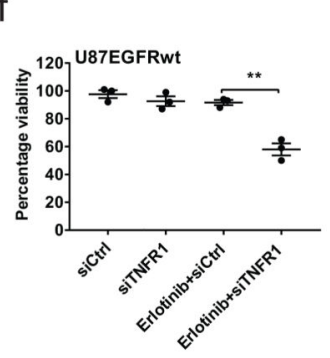

j
C

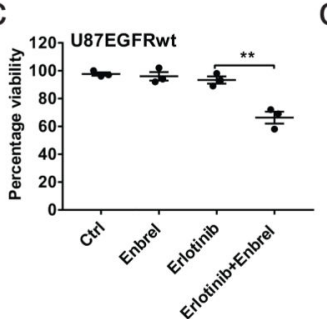

g

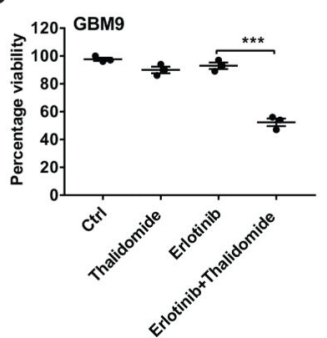

$\mathrm{k}$ d

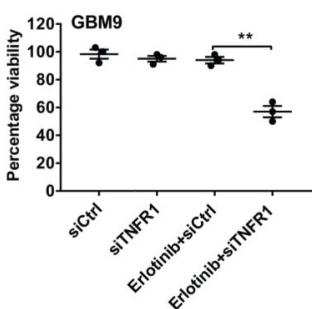

$\mathrm{h}$

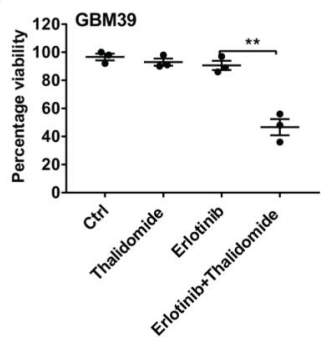

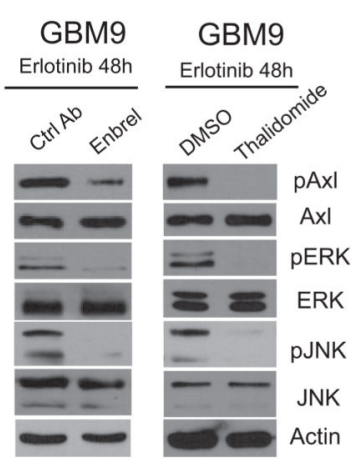

m

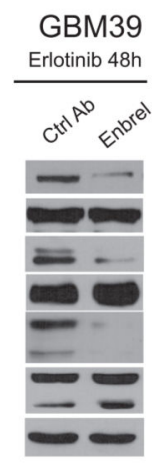

GBM39

Erlotinib 48h

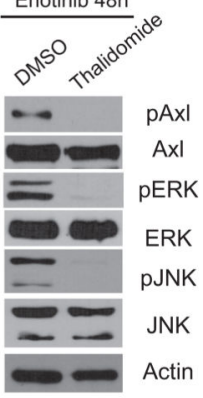

n

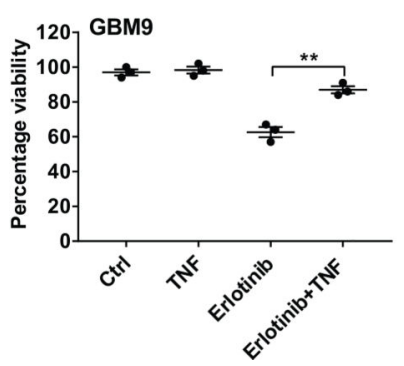

Figure 6. TNF inhibition sensitizes glioma cells to EGFR inhibition

(a-b) AlamarBlue cell viability assay in GBM9 or GBM39 neurospheres. Enbrel (100 nM) sensitizes cells to EGFR inhibition with erlotinib. Enbrel and erlotinib were added to GBM9 or GBM39 neurospheres concurrently and AlamarBlue assay was done after 72h. (a) Erlotinib vs Erlotinib+Enbrel: $P=0.0027, \mathrm{t}=6.59$, d.f. $=4$. (b) Erlotinib vs erlotinib+enbrel: $P$ $=0.0044, \mathrm{t}=6.59$, d.f. $=4$. (c) A similar experiment was performed in U87EGFRwt cells. Erlotinib vs Erlotinib+Enbrel: $P=0.0056, \mathrm{t}=5.41$, d.f. $=4$. $(\mathrm{d}-\mathrm{e})$ TNFR1 was silenced using siRNA in GBM9 and GBM39 cells and cells were exposed to erlotinib for $72 \mathrm{~h}$ in stem cell 
medium without EGF for $72 \mathrm{~h}$ followed by Alamarblue Assay. (d) Erlotinib+siCtrl vs erlotinib+siTNFR1: $P=0.0014, \mathrm{t}=7.95$, d.f.=4. (e) Erlotinib+siCtrl vs erlotinib+siTNFR1: $P$ $=0.0041, \mathrm{t}=5.90$, d.f.=4. (f) A similar experiment was done in U87EGFRwt cells. Erlotinib +siCtrl vs erlotinib+siTNFR1: $P=0.0021, \mathrm{t}=7.11$, d.f.=4. $(\mathrm{g}-\mathrm{i})$ Thalidomide sensitizes GBM9 and GBM39 cells to EGFR inhibition with erlotinib. Thalidomide $(1 \mu \mathrm{M})$ and erlotinib were added to GBM9 and GBM39 neurospheres (100nM) or U87EGFRwt cells (1uM) concurrently and AlamarBlue assay was done after $72 \mathrm{~h}$. (g) Erlotinib vs erlotinib +thalidomide: $P=0.0030, \mathrm{t}=6.42$, d.f. $=4$. (h) Erlotinib vs erlotinib+thalidomide: $P=0.0027$, $\mathrm{t}=6.59$, d.f. $=4$. (i) Erlotinib vs erlotinib+thalidomide: $P=0.0013$, $\mathrm{t}=8.11$, d.f. $=4$. $(\mathrm{j}-\mathrm{k})$ Enbrel or thalidomide block erlotinib induced activation of JNK, Axl and ERK in GBM9 and GBM39 neurospheres as shown in the Western blot. (1) A similar experiment was conducted in U87EGFRwt cells. Western blots shown in $\mathrm{j}-1$ are representative of at least three independent replicates. Full-length blots are presented in Supplementary Figure 14. (m-n) show that exogenous TNF protects GBM9 and GBM39 neurospheres from erlotinib induced cell death. TNF $(1 \mathrm{ng} / \mathrm{ml})$ and erlotinib $(1 \mu \mathrm{M})$ were added to cells concurrently and AlamarBlue cell viability assay was done after $72 \mathrm{~h}$. (m) Erlotinib vs erlotinib+TNF: $P$ $=0.0018, \mathrm{t}=7.41$, d.f.=4. (n) Erlotinib vs erlotinib+TNF: $P=0.0087, \mathrm{t}=4.79$, d.f. $=4$. Data are presented as mean \pm s.e.m; $* * P<0.01, * * * P<0.001$ from two-tailed unpaired Student's t-test ( $n=3$ biologically independent experimental replicates). 

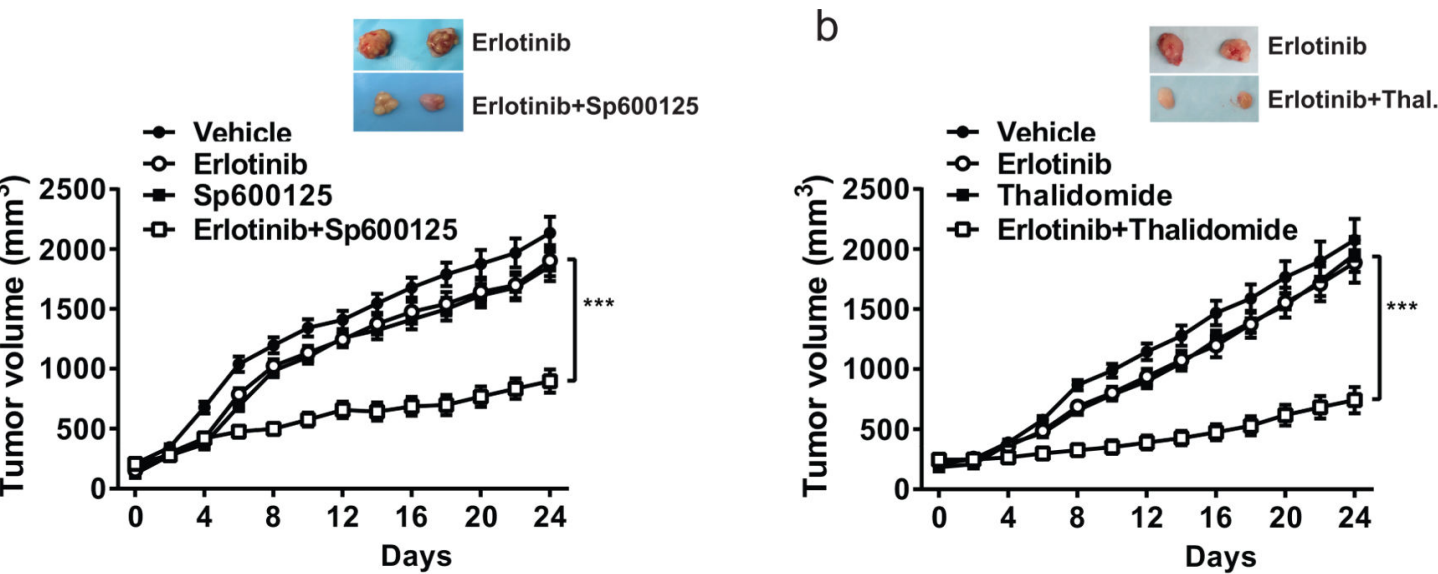

C

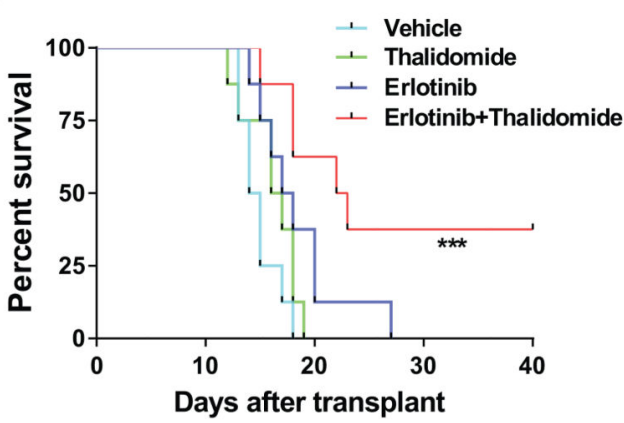

d

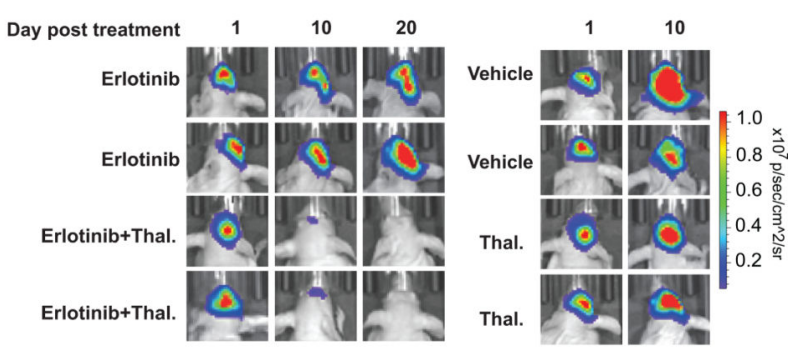

e

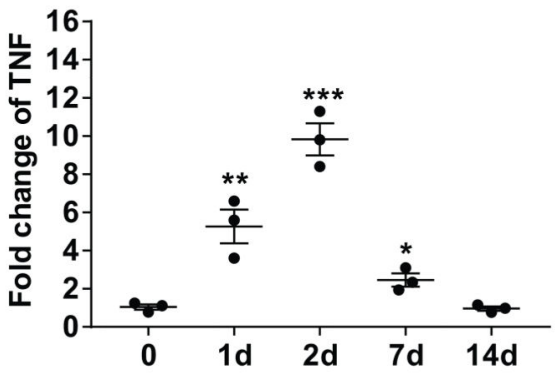

f

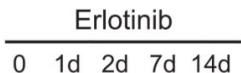

g
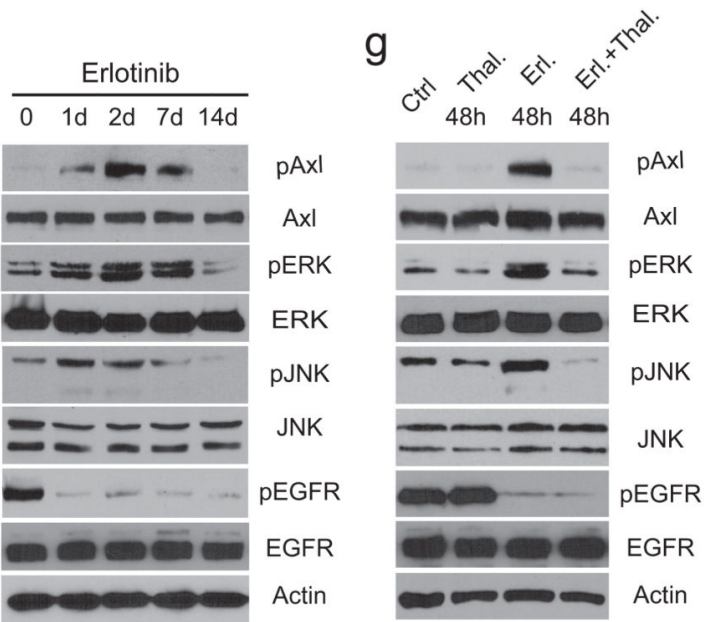

Figure 7. JNK or TNF inhibition sensitizes mouse tumors to EGFR inhibition in vivo

(a) Treatment of subcutaneous tumors with a combination of erlotinib and SP600125. The tumor growth did not decrease in mice treated erlotinib or SP600125 alone, whereas the combination of erlotinib and SP600125 was found to decrease tumor growth significantly. Unpaired t-test, erlotinib vs erlotinib+SP600125: $P=0.0003$, $\mathrm{t}=4.70$, d.f. $=14$, $* * * P<0.001$.

(b) Treatment of subcutaneous tumors with a combination of erlotinib and thalidomide. The tumor growth did not decrease in mice treated erlotinib $(50 \mathrm{mg} / \mathrm{kg})$ or thalidomide $(150 \mathrm{mg} / \mathrm{kg})$ alone, whereas the combination of erlotinib and thalidomide was found to 
decrease tumor growth significantly. Unpaired t-test, Erlotinib vs erlotinib+thalidomide: $* * * * P<0.0001, \mathrm{t}=6.1$, d.f.=14. (c) Combined treatment of erlotinib and thalidomide prolonged survival and suppressed tumor growth in an orthotopic model. Kaplan-Maier survival curves were calculated using GraphPad Prism 7. Statistical significance verified by the log rank test, $P=0.0008$, $* * * P<0.001$. (d) Representative bioluminescence images from erlotinib and erlotinib plus thalidomide group at day 1,10 and 20 post-treatment. Since all the mice in vehicle and thalidomide group died within 20 days after transplant, images at day 20 post-treatment were not available. (e) Time course of TNF upregulation in mouse tumors exposed to erlotinib $50 \mathrm{mg} / \mathrm{kg}$ for the indicated time points $(\mathrm{n}=3)$. Tumors were removed following erlotinib exposure followed by TNF ELISA on protein extracts. 0 vs $1 \mathrm{~d}$ : $P=0.0091$, $\mathrm{t}=4.73$, d.f. $=4 ; 0$ vs $2 \mathrm{~d}: P=0.0005$, $\mathrm{t}=10.36$, d.f. $=4 ; 0$ vs $7 \mathrm{~d}: P=0.0181$, $\mathrm{t}=3.86$, d.f. $=4$. Data are presented as mean \pm s.e.m; $* P<0.05$, $* * P<0.01$, $* * * P<0.001$ from a twotailed unpaired Student's t-test. (f) Shows signal transduction in intracranial tumors exposed to erlotinib $(50 \mathrm{mg} / \mathrm{kg}$ ) for the indicated time points. (g) Western blots of intracranial tumor lysates obtained from erltinib and/or thalidomide treated mice. The animals without treatment were considered as $\mathrm{Ctrl}$ (control, 0-day treatment). Western blots shown in $\mathrm{f}$ and $\mathrm{g}$ are representative of three independent replicates. Full-length blots are presented in Supplementary Figure 14. 